\title{
Identifying the Extent of Completeness of Query Answers over Partially Complete Databases
}

\author{
Simon Razniewski \\ Free University of \\ Bozen-Bolzano \\ razniewski@inf.unibz.it
}

\author{
Flip Korn \\ Google Research \\ flip@google.com
}

\author{
Werner Nutt \\ Free University of \\ Bozen-Bolzano \\ nutt@inf.unibz.it
}

\author{
Divesh Srivastava \\ AT\&T Labs-Research \\ divesh@research.att.com
}

\begin{abstract}
In many applications including loosely coupled cloud databases, collaborative editing and network monitoring, data from multiple sources is regularly used for query answering. For reasons such as system failures, insufficient author knowledge or network issues, data may be temporarily unavailable or generally nonexistent. Hence, not all data needed for query answering may be available.

In this paper, we propose a natural class of completeness patterns, expressed by selections on database tables, to specify complete parts of database tables. We then show how to adapt the operators of relational algebra so that they manipulate these completeness patterns to compute completeness patterns pertaining to query answers. Our proposed algebra is computationally sound and complete with respect to the information that the patterns provide. We show that stronger completeness patterns can be obtained by considering not only the schema but also the database instance and we extend the algebra to take into account this additional information. We develop novel techniques to efficiently implement the computation of completeness patterns on query answers and demonstrate their scalability on real data.
\end{abstract}

\section{INTRODUCTION}

In many applications, data from multiple sources (tables, sites, authors, measurement systems, etc.) is used in query answering. Examples are loosely coupled cloud databases such as AzureDB [16 15], crowd-sourced collaborative projects such as Wikipedia, and warehouses collecting monitoring data such as Darkstar [14].

In all these scenarios, data may be incomplete for a variety of reasons. In the first case of cloud databases, incompleteness occurs mainly due to network delays or the failure of data nodes, which may render data to be temporarily unavailable. In the second case, the open nature of collaborative databases makes it possible for anyone to enter nearly any information, and incompleteness arises due to insufficient or biased author knowledge. In the third case of network data warehouses, partial integration of heterogeneous source feeds or operational failures of reporting systems may lead to the data warehouse having only partially complete information.

Permission to make digital or hard copies of all or part of this work for personal or classroom use is granted without fee provided that copies are not made or distributed for profit or commercial advantage and that copies bear this notice and the full citation on the first page. Copyrights for components of this work owned by others than ACM must be honored. Abstracting with credit is permitted. To copy otherwise, or republish, to post on servers or to redistribute to lists, requires prior specific permission and/or a fee. Request permissions from permissions@acm.org.

SIGMOD'15, May 31-June 4, 2015, Melbourne, Victoria, Australia

Copyright is held by the owner/author(s). Publication rights licensed to ACM.

ACM 978-1-4503-2758-9/15/05 ...\$15.00.

http://dx.doi.org/10.1145/2723372.2750544.
To address incompleteness in these scenarios, many different mechanisms have been investigated. For cloud databases, Lang et al. 16 15] identified the sources of incompleteness and traced how it is propagated through a query plan. For Wikipedia, a template has been introduced that allows lists to be labeled as complete. For data streams feeding network databases, it was proposed to deliver data only after so-called punctuations have arrived, that is, checkpoints that enable one to conclude that all data up to a certain timepoint has been received [19, 12]. Further, the differences between these scenarios has resulted in different languages used to specify completeness. While the first approach uses units of computation (shards, columns, tables), the second uses natural-language statements, and the third uses temporal selections on data streams.

Despite these differences, a commonality across these scenarios is that completeness specifications of parts of databases are given. Also, in all three scenarios, knowledge about which parts of the query results are complete can help users understand whether their information needs can be satisfied with the available data. In this paper, we focus on this problem of using completeness information for parts of a database to annotate query answers with information about which parts of the query answers are complete. If the presented results do not meet user expectations, they can then try to consult specific additional data sources, use historical data/statistical approaches to estimate missing data, or reformulate their queries. This is preferable to users incorrectly assuming that their query results are complete, or alternatively that no part of their query results is complete.

The setting discussed here differs from the one commonly called incomplete databases (for a recent survey, see e.g. [18]). There, one focuses on the meaning and consequences of null values, that is, individual cell values in known records that are missing or incomplete. In contrast, we investigate the impact on query results of entire database records that may be missing.

Network Data Warehouse Use Case. We consider a simplified database $D_{\text {maint }}$ of a network provider, for illustrative purposes. The database schema contains the following three tables:

- Warnings(day, week, ID, message),

- Maintenance(ID, responsible, reason),

- Teams(name, specialization).

The first table stores the warnings received from network elements, identified by an ID, together with a time stamp. The second stores the set of network elements that are currently in maintenance, together with the responsible teams and the reasons for maintenance. The third stores the maintenance teams together with their specialization. We sometimes refer to these tables using only the first letter of their name, i.e., $W, M$, and $T$. 


\begin{tabular}{c|c|c|c|c|}
\cline { 2 - 5 } \multicolumn{1}{c|}{} & \multicolumn{4}{|c|}{ Warnings } \\
\cline { 2 - 5 } & day & week & ID & message \\
\cline { 2 - 5 }$r_{1}$ & Mon & 1 & tw37 & high voltage \\
\cline { 2 - 5 }$r_{2}$ & Fri & 1 & tw37 & high voltage \\
\cline { 2 - 5 }$r_{3}$ & Wed & 2 & tw37 & overheated \\
\cline { 2 - 5 }$r_{4}$ & Tue & 1 & tw59 & auto restart \\
\cline { 2 - 5 }$r_{5}$ & Fri & 1 & tw59 & overheat \\
\cline { 2 - 5 }$r_{6}$ & Mon & 2 & tw83 & high voltage \\
\cline { 2 - 5 }$r_{7}$ & Tue & 2 & tw83 & auto restart \\
$p_{1}$ & $*$ & 1 & $*$ & $*$ \\
$p_{2}$ & Mon & 2 & $*$ & $*$ \\
$n_{3} p_{3}$ & Wed & 2 & $*$ & $*$ \\
\cline { 2 - 5 } & & & &
\end{tabular}

\begin{tabular}{c|c|c|c|}
\cline { 2 - 4 } \multicolumn{1}{c|}{} & \multicolumn{3}{c|}{ Maintenance } \\
\cline { 2 - 4 } & ID & responsible & reason \\
\cline { 2 - 4 }$r_{8}$ & tw37 & $\mathrm{A}$ & disk failure \\
\cline { 2 - 4 }$r_{9}$ & $\mathrm{tw} 59$ & $\mathrm{D}$ & software crash \\
\cline { 2 - 4 }$r_{10}$ & $\mathrm{tw} 83$ & $\mathrm{~B}$ & unknown \\
\cline { 2 - 4 }$r_{11}$ & $\mathrm{tw} 140$ & $\mathrm{C}$ & update failure \\
\cline { 2 - 4 }$r_{12}$ & $\mathrm{tw} 140$ & $\mathrm{C}$ & network error \\
\cline { 2 - 4 }$p_{4}$ & $*$ & $\mathrm{~A}$ & $*$ \\
$p_{5}$ & $*$ & $\mathrm{~B}$ & $*$ \\
$p_{6}$ & $*$ & $\mathrm{C}$ & $*$ \\
\cline { 2 - 4 } & & \multicolumn{3}{c}{}
\end{tabular}

\begin{tabular}{c|c|c|}
\cline { 2 - 3 } \multicolumn{1}{c|}{} & \multicolumn{2}{|c|}{ Teams } \\
& name & specialization \\
\cline { 2 - 3 }$r_{13}$ & $\mathrm{~A}$ & hardware \\
\cline { 2 - 3 }$r_{14}$ & $\mathrm{~B}$ & hardware \\
\cline { 2 - 3 }$r_{15}$ & $\mathrm{C}$ & network \\
\cline { 2 - 3 }$r_{16}$ & $\mathrm{C}$ & software \\
\cline { 2 - 3 }$r_{17}$ & $\mathrm{D}$ & network \\
\cline { 2 - 3 }$p_{7}$ & $*$ & $*$ \\
\cline { 2 - 3 } & &
\end{tabular}

Table 1: Database $D_{\text {maint }}$ annotated with completeness information.

A sample database using this schema is depicted in Table 1 Each table contains in addition to the actual records (rows labeled with $r$ ) also a set of completeness patterns (rows labeled with $p$ ).

Suppose that warnings are loaded daily from an operational system. Suppose the current date is Thursday, week 2. The reports from last week (week 1) have all been loaded, and from this week, so far the reports for Monday and Wednesday have been loaded, while not all the ones for Tuesday have arrived yet. Then the completeness of the data for week 1, and for Monday and Wednesday of week 2 , are expressed by the completeness patterns $(*, 1, *, *)$, (Mon, $2, *, *)$, (Wed, $2, *, *)$, which appear as patterns $p_{1}$ to $p_{3}$ in Table 1. Intuitively, these patterns assert that all possible records that describe real-world facts and that match these patterns (with "*" being a wildcard), are present in the database.

Suppose also that we have information about all network elements currently in maintenance with teams A, B and C, because their local systems automatically export data to the central database, while others do not. This information is represented by the completeness patterns $p_{4}$ to $p_{6}$ in Table 1 .

We also know all maintenance teams in the company, with their specializations. This is pattern $p_{7}$.

Suppose now we are interested in getting all warnings in week 2 for all elements that are in maintenance with a hardware team being responsible. This information can be obtained via the following SQL query $Q_{h w}$ :

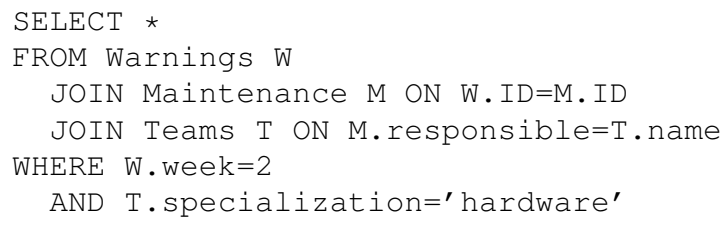

This query can be expressed by different algebraic expressions, for instance the following:

$$
\begin{aligned}
\sigma_{\text {week=2 }}(W) & \bowtie_{W . I D=M . I D} \\
& \left(M \bowtie_{M . \text { resp }=T \text {.name }} \sigma_{\text {spec }=" h w "}(T)\right) .
\end{aligned}
$$

When evaluating this expression over the database $D_{\text {maint }}$ we get the result shown in the first three rows in Table 3 But can we also use the completeness information that we have about the database to learn about the completeness of the query result?

Let us look at the example of the first selection operation in the expression shown above, that is, $\sigma_{\text {week }=2}(W)$. The pattern $p_{1}=$ $(*, 1, *, *)$ does not tell anything about the result of this selection because it talks about completeness of week 1 only.

\begin{tabular}{|c|c|c|c|}
\hline \multicolumn{4}{|c|}{$\sigma_{\text {week=2 }}$ (Warnings) } \\
day & week & ID & message \\
\hline \hline Wed & 2 & tw37 & overheated \\
\hline Mon & 2 & tw83 & high voltage \\
\hline Tue & 2 & tw83 & auto restart \\
\hline \hline Mon & $*$ & $*$ & $*$ \\
\hline Wed & $*$ & $*$ & $*$ \\
\hline
\end{tabular}

Table 2: Intermediate data and metadata after the selection operation $\sigma_{\text {week }=2}$ (Warnings).

The pattern $p_{2}$, however, which is (Mon, $\left.2, *, *\right)$, does tell us something about the result. Since we are selecting data only from week 2 , and $p_{2}$ says that all data from Monday week 2 is there, we can infer that the result will be complete for all data on Monday. Thus, we can infer that the pattern (Mon, $*, *, *)$ holds over the result. The same holds for the pattern $p_{3}$. We summarize this information in Table 2

We can proceed similarly for the selection $\sigma_{\text {spec }=\text { "hw" }}(T)$, which preserves the pattern $(*, *)$. Subsequently joining the completeness patterns analogously to the base data, with the wildcard matching any constants, we arrive at the conclusion that the query result is complete for teams A, B and C on Monday and Wednesday. This can be represented using completeness patterns as shown in Table 3 Note that these conclusions have been drawn by applying algebraic operations only to the patterns $p_{1}$ to $p_{7}$.

Taking into account the interplay of query and state of the database, more can be concluded: Inspecting the database, one observes that teams A and B are the only known hardware teams, and that due to the completeness of the Teams table, no other hardware teams can exist. Since the query asks only for hardware teams, it is therefore possible to summarize the six completeness patterns for the teams $\mathrm{A}, \mathrm{B}$ and $\mathrm{C}$ into just two patterns where $\mathrm{A}, \mathrm{B}$ and $\mathrm{C}$ have been replaced by a "*”. The result can be seen in Table 5 We will later refer to this summarization as pattern promotion.

An end user would therefore know that on Monday and Wednesday, the retrieved warnings for tw 37 and tw 83 are really the only ones that occurred. In contrast, for Tuesday, the user can see that a warning for tw83 occurred, but there is no assurance that this is the only warning that occurred on that day. To assure this, the user would need to either load the data from Tuesday manually, or consult the network admins to verify that no other warnings occurred on that day.

Previous approaches (see Section 2 would only be able to say that the query $Q_{h w}$ is not complete over the database $D_{\text {maint }}$. 


\begin{tabular}{|c|c|c|c|c|c|c|c|c|}
\hline$W$.day & $W$. week & $W . I D$ & $W . m e s s a g e$ & $M . I D$ & $M$. responsible & $M$. reason & $T$. name & $T$.specialization \\
\hline \hline Wed & 2 & tw37 & overheated & tw37 & A & disk failure & A & hardware \\
\hline Mon & 2 & tw83 & high voltage & tw83 & B & unknown & B & hardware \\
\hline Tue & 2 & tw83 & auto restart & tw83 & B & unknown & B & hardware \\
\hline \hline Mon & $*$ & $*$ & $*$ & $*$ & A & $*$ & A & $*$ \\
\hline Mon & $*$ & $*$ & $*$ & $*$ & B & $*$ & B & $*$ \\
\hline Mon & $*$ & $*$ & $*$ & $*$ & C & $*$ & C & $*$ \\
\hline Wed & $*$ & $*$ & $*$ & $*$ & A & $*$ & A & $*$ \\
\hline Wed & $*$ & $*$ & $*$ & $*$ & B & $*$ & B & $*$ \\
\hline Wed & $*$ & $*$ & $*$ & $*$ & C & $*$ & C & $*$ \\
\hline
\end{tabular}

Table 3: Result of the query $Q_{h w}$ over the database $D_{\text {maint }}$ annotated with completeness information.

\begin{tabular}{|c|c|c|c|}
\hline name & country & $\begin{array}{l}\text { City } \\
\text { state }\end{array}$ & county \\
\hline$*$ & USA & Virginia & * \\
\hline$*$ & Germany & $*$ & * \\
\hline$*$ & Ukraine & $*$ & $*$ \\
\hline * & Bulgaria & * & * \\
\hline$*$ & USA & New York & $*$ \\
\hline$*$ & UK & Carmarthenshire & * \\
\hline$*$ & USA & West Virginia & Hampshire County \\
\hline * & Czech & Moravian-Silesia & Novy Jicin \\
\hline
\end{tabular}

Table 4: Completeness statements for cities on Wikipedia

Wikipedia Use Case. Wikipedia is an extreme example of a crowd-sourced database, as a vast number of authors are contributing to articles. As a consequence, completeness of the data is a big issue 11 To specify completeness of data in various contexts such as enumerated lists, authors use either natural language statements, e.g. "This is a complete list of cities in the Commonwealth of Virginia in the United States" 2 or a template to mark lists as complete ${ }^{3}$ Currently, $\sim 500$ pages use the template and $\sim 5000$ pages use natural language expressions to state completeness.

Suppose we want to count the number of cities in certain countries. In the spirit of DBpedia [3], we can scrape the data from Wikipedia to obtain lists of cities in various countries. But what quality guarantees do we find for this data? Table 4 shows the completeness statements that we found for cities on Wikipedia. If we were e.g. to count the number of cities in Bulgaria based on the Wikipedia data, then we can use the guarantee given by some author that Wikipedia really contains all those cities. In contrast, for France there is no completeness statement (and indeed the relevant article does not include cities with small populations). Knowledge of which countries are associated with completeness statements, and which ones are not, can lead users to query specific additional sources, such as the Mondial database or the CIA world factbook, as needed.

Contributions. In the rest of this paper, we discuss how the intuitive conclusions above can be formalized and automated. Our contributions are the following:

\footnotetext{
${ }^{1}$ Correctness is an issue as well, but we ignore this for the moment. 2 http://en.wikipedia.org/wiki/List_of_ cities_in_Virqinia

3 http://en.wikipedia.org/wiki/Template: Complete_list
}

- We introduce a natural class of completeness patterns that correspond to selections on database tables and can be expressed in the same schema as the normal data.

- We define a pattern algebra that, given completeness patterns as input, provides metadata as output to identify the extent of completeness of the query answer over a partially complete database. This metadata is computed similarly to the way the query answer itself is computed and can be executed almost as efficiently.

- We extend this algebra to additionally take into account the database instance, thus allowing one to compute more general completeness patterns (i.e., stronger assertions), but potentially requiring computation time exponential in the size of the data.

- We develop techniques to efficiently implement the algebras presented and show their scalability on real data. Our experiments show that considering the database instance does indeed lead to stronger completeness assertions while avoiding exponential blow-up in practice.

Outline. This paper is structured as follows. In Section 2 we discuss related work, in Section 3 we formalize partially complete databases and completeness patterns, in Section 4 we present an algebra for completeness patterns, in Section 5 we extend this algebra to consider the state of the database instance, and discuss practical aspects of our framework in Section 6

\section{RELATED WORK}

The classical paradigm for databases is the closed-world assumption [23], which asserts that the information in the database is complete. For data integration and on the Semantic Web, often the open-world assumption is preferred [1], where nothing is assumed about the completeness of the database. Open and closed-world semantics were first discussed by Reiter in [23], where he formalized that negation as failure [6] corresponds to standard negation under the closed world assumption.

Scenarios as the one discussed in our motivating examples, however, require a middle ground. In such situations, parts of the database known to be complete are considered closed-world, while the incomplete parts are open-world. This intermediate paradigm, called the partial (or, local [7]) closed-world assumption, has not received as much attention so far.

The first formalization of this setting was by Motro [21], who showed how to derive the completeness of a query answer given statements about the completeness of other query answers. Subsequently, Levy extended this idea by introducing table completeness (TC) statements [17], which are similar to tuple-generating dependencies, and showed how to derive query answer complete- 


\begin{tabular}{|c|c|c|c|c|c|c|c|c|}
\hline$W$.day & $W$.week & $W$.ID & $W$.message & $M$.ID & $M$.responsible & $M$.reason & $T$.name & $T$.specialization \\
\hline \hline Wed & 2 & tw37 & overheated & tw37 & A & disk failure & A & hardware \\
\hline Mon & 2 & tw83 & high voltage & tw83 & B & unknown & B & hardware \\
\hline Tue & 2 & tw83 & auto restart & tw83 & B & unknown & B & hardware \\
\hline \hline Mon & $*$ & $*$ & $*$ & $*$ & $*$ & $*$ & $*$ & $*$ \\
\hline Wed & $*$ & $*$ & $*$ & $*$ & $*$ & $*$ & $*$ & $*$ \\
\hline
\end{tabular}

Table 5: Result of the query $Q_{h w}$ over the database $D_{\text {maint }}$ with summarized completeness information

ness from them. The completeness patterns used in this paper are exactly the intersection of the statements introduced by Motro and Levy. While Levy's statements allow one to express the completeness of a table in terms of other, incomplete tables, Motro's statements assume that all tables used in a statement are complete. The intersection of these two is therefore the set of all statements that do not use additional tables.

Other work, such as Denecker et al. [9], Cortes-Calabuig et al. [7] and Razniewski and Nutt [22], looked into reasoning about query completeness using the database instance. However, they used more expressive languages for their completeness descriptions and/or queries and, thus, suffer from high complexity (coNP-data complexity or $\Pi_{2}^{P}$-query complexity). More importantly, the previous work only studied the decision problem of whether the query results are fully complete.

Fan and Geerts [10,11] investigated a related problem of completeness assessment in the presence of a database instance. However, they make the assumption that a master ("upper-bound") database instance is given to be able to determine which tuples are missing, which may not always be available.

Closer in spirit to our approach is the work by Lang et al. [16], who address the problem of characterizing partial results computed by query plans when some data may be unavailable. Their key contributions are: (1) a taxonomy for classifying query result semantics based on two properties of the partial result: cardinality and correctness; according to cardinality, the result may be missing some tuples or may have extra tuples; according to correctness, the result may be credible or non-credible; (2) an identification of four models, with different granularities of analysis: query, operator, column and partition; and (3) a discussion of how the partial result semantics can be propagated through various operators in a query plan. A recent paper by Lang et al. [15] additionally allows users to control the cost of a query returning partial results by defining the sorts of partial results that are acceptable. In terms of the classification by Lang et al., we explore the cardinality dimension (completeness/incompleteness), for the (finest) partition granularity, in much greater technical depth. Our key contributions, which distinguish us from [16], are: (1) a natural class of completeness patterns that correspond to selections on database tables; (2) pattern algebras that allow us to formally reason over completeness patterns and the database instance as input, to compute completeness patterns on the query output - in particular, our techniques can use the database instance to infer stronger completeness patterns on query results compared to the techniques described in |16]; and (3) techniques to efficiently implement the algebras presented, and an empirical evaluation of their scalability in practice. Thus, the technical contributions of the two papers are complementary.

Punctuations, checkpoints that allow one to conclude that all data up to a certain timepoint has been received, are used in data streams as a way to assert completeness in a time interval [19. 12]. While they are most often used to assert temporal completeness, they have a similar flavor to our completeness patterns. However, existing work has not studied how to use punctuations to determine which portions of query answers are complete.

Data cleaning, the process of detecting and correcting errors, inconsistencies, duplicates and other problems related to data correctness, has received considerable attention in the past $[13,4$ 26]. Regarding incompleteness, extensive work has been done on statistical approaches to missing value imputation [24 2]. The difference to our problem is that in missing value imputation, one is already aware of the location of the incompleteness.

Finally, we note that our setting is different from what in the literature is commonly called incomplete databases (for a recent survey, see e.g. [18]). While this setting allows also for missing records, in most of this work, implications on query answers of null values in existing records is studied, such as providing certain answers, where query answers over all possible completions of an incomplete database are provided, and possible answers, where answers over at least one such completion are provided [1]. In contrast, we investigate the problem of how missing records in the database lead to missing records in the query answer, and if so, from which parts of the query answer.

\section{DATABASE AND QUERY COMPLETENESS PATTERNS}

In this section we review database terminology and notation and introduce the framework for completeness assessment.

We first introduce databases and the query language we are using in this paper, then formalize completeness patterns, the semantics of completeness patterns, and the notion of entailed completeness patterns for query answers.

\subsection{Databases and Queries}

A relation schema is a sequence of attributes. A database schema is a set of table names, each with a relation schema. If table $R$ has attributes $A_{1}, \ldots, A_{n}$, we say that $n$ is the arity of $R$. We often write a tuple $t$ for $R$ as $t=\left(d_{1}, \ldots, d_{n}\right)$, where $d_{1}, \ldots, d_{n}$ are constants. We interchangeably refer to $d_{i}$, the value of $t$ for attribute $A_{i}$, as $t\left[A_{i}\right]$ or simply $t[i]$. Given a fixed schema, a database instance $D$ stores for each table name a finite bag (= multiset) of tuples for that table. The bag of tuples in $D$ for table $R$ is denoted as $R(D)$. If schema and instance are clear from the context, we simply speak of a database.

An example of a database is $D_{\text {maint }}$ as shown in Table 1 while ignoring the rows with $p_{i}$.

As queries we consider those that can be expressed using the relational algebra operations select, project and (equi)join (SPJ), which correspond to single-block queries in SQL. To ease the exposition, we do not consider renaming in our presentation.

As in SQL, we assume bag semantics for both databases and queries, that is, both can contain the same row multiple times. When discussing generic properties of a query, we use the letter $Q$. We use the letter $E$ if the specific structure of the expression is im- 
portant. With $Q(D)$ and $E(D)$ we denote their evaluation over a database $D$.

\subsection{Completeness Patterns}

We now want to formalize the meaning of completeness patterns such as rows $p_{1}$ to $p_{7}$ in Table 1 , and the bottom six rows in Table 3 A completeness pattern is a tuple composed of constants or the wildcard symbol " $*$ ". As patterns like those in Table 1 are expressed over database tables, we call them base completeness patterns. As the patterns in Table 3 refer to a query result, we call them query completeness patterns.

Definition 1 (BASE Completeness Pattern). $A$ base completeness pattern for a table $R$ is a pair $(p, R)$ where $p$ is a completeness pattern with the same arity as $R$.

The relation $R$ is dropped if it is clear from the context.

Example 1. Assume we want to say that the Maintenance table is complete for all elements maintained by team A. Then this can be expressed by the base completeness pattern $(*, \mathrm{~A}, *)$, for Maintenance, which is the pattern $p_{4}$ in Table 1

A set of completeness patterns for a table can be stored in a corresponding metadata table, which has the same attributes as the data table. In the following, we assume that in a database each table has an associated metadata table.

Intuitively, a base completeness pattern says that all rows of a certain kind, namely those subsumed by the pattern, are present in the table and no rows of this form are missing. In this way, they create a middle ground between closed and open-world assumption.

A pattern $p_{1}$ subsumes a pattern $p_{2}$ if for every position $i$ it holds that $p_{1}[i]=p_{2}[i]$ or $p_{1}[i]=*$. In other words, $p_{1}$ and $p_{2}$ are the same except that $p_{1}$ can contain additional $*$-values. A pattern can also subsume a data tuple, since tuples are a special case of patterns.

Consider a database $D$. A second database $D^{c}$, with the same schema, contains $D$, written $D \subseteq D^{c}$, if $R(D) \subseteq R\left(D^{c}\right)$ for all relations $R$ in the schema. Intuitively, a database $D^{c}$ containing $D$ corresponds to a possible state of the real world, about which $D$ has only limited information.

We say that $D^{c}$ satisfies the completeness pattern $(p, R)$ wrt. $D$ if $D \subseteq D^{c}$ and all tuples in $R\left(D^{c}\right)$ that are subsumed by $p$ are also in $R(D)$. While in general, we do not know what is all the data that holds in reality, completeness patterns restrict the possible complete databases to the satisfying ones. Although completeness patterns in general still allow infinitely many possible states of the real world, all these possible states contain the same information in the parts asserted to be complete.

DEFINITION 2 (CANDidATE SET). Let D be a database and $\mathcal{P}$ be a set of base completeness patterns that are annotated with their respective relations. A database $D^{c}$ with the same schema is called $a$ candidate completion for $D$ and $\mathcal{P}$ if (1) $D \subseteq D^{c}$ and (2) $D^{c}$ satisfies all patterns in $\mathcal{P}$ wrt. $D$.

The set of all candidate completions is called the candidate set, written $\operatorname{Cand}^{\mathcal{P}}(D)$.

Example 2. Consider again the database $D_{\text {maint }}$ from Table 1 Because of pattern $p_{3}$, which expresses that all warnings from Wednesday of week 2 are already in $D_{\text {maint }}$, a database $D^{c}$ that contains the fact Warnings(Wed, 2, tw37, highvoltage) would not be a candidate.

On the other hand, a database $D^{c}$ that additionally contains the fact Warnings(Tue, 2, tw37, highvoltage) would be a candidate, since it is not subsumed by any pattern for Warnings.
We remark that base completeness patterns capture exactly the intersection of the statements defined in [21] and [17], as they do not contain any join conditions, but are purely selections on tables. We chose the class of completeness patterns because, in contrast to the previously used statements, base completeness patterns can be expressed as rows in the same schema as the data.

We now define completeness patterns for queries, such as the bottom six rows in Table 3 Completeness patterns for queries are patterns paired with query expressions, for parts of which they assert completeness.

For a query $Q$ with result schema $A_{1}, \ldots, A_{n}$ we use the shorthand " $\operatorname{attr}(Q)=p$ " to denote the condition " $A_{1}=p_{1} \wedge \cdots \wedge$ $A_{n}=p_{n}$ ", where a conjunct " $A_{i}=*$ " simplifies to true.

Definition 3 (Query Completeness Pattern). A query completeness pattern for a query $Q$ is a pair $(p, Q)$, where $p$ is a pattern that conforms to the result schema of $Q$. The associated query of $(p, Q)$ is $Q_{p}:=\sigma_{a t t r}(Q)=p(Q)$.

$A$ database $D^{c}$ satisfies $(p, Q)$ wrt. $D$, if $Q_{p}\left(D^{c}\right)=Q_{p}(D)$.

Intuitively, a completion $D^{c} \supseteq D$ satisfies $(p, Q)$ if all answer tuples $t \in Q\left(D^{c}\right)$ that match $p$ are already present in $Q(D)$.

Note that a tuple $t \in R\left(D^{c}\right)$ is subsumed by a pattern $p$ iff $t \in \sigma_{\text {attr }(R)=p}\left(R\left(D^{c}\right)\right)$. Thus, $D^{c}$ satisfies the base pattern $(p, R)$ wrt. $D$ iff $\sigma_{a t t r(R)=p}\left(R\left(D^{c}\right)\right) \subseteq R(D)$. In other words, satisfaction of base patterns is a special case of query patterns if we view base relations as queries that return the content of a database table.

Completeness patterns can be compared wrt. generality. We say that $p_{1}$ is more general than $p_{2}$ if every tuple subsumed by $p_{2}$ is also subsumed by $p_{1}$. It is straightforward to see that $p_{1}$ is more general than $p_{2}$ if $p_{1}$ subsumes $p_{2}$.

For example, the pattern $(*, A, *)$ for the Maintenance table subsumes $(*, A$, unknown). At the same time, every tuple subsumed by the second pattern is also subsumed by the first.

Let $P$ be a set of patterns over the same schema. Clearly, subsumption is a partial order on $P$. A pattern $p_{0} \in P$ is maximal in $P$ if there is no pattern in $P$ that strictly subsumes $p_{0}$. Clearly, every element in $P$ is subsumed by some element that is maximal in $P$.

We say that $P$ is minimal (wrt. subsumption) if no element is strictly subsumed by another element. We are interested in minimal sets of patterns because they do not contain any redundant patterns.

It is straightforward to see that $P$ is minimal if and only if all its elements are maximal in $P$. Thus, to minimize a set $P$ of patterns, one has to eliminate from $P$ all non-maximal elements.

\subsection{Problem Statement}

We are investigating how to compute query completeness patterns (Def. 33) from base completeness patterns (Def.11) so that the results satisfy the following entailment property.

Definition 4 (ENTAILMENT). Let $D$ be a database and $\mathcal{P}$ be a set of base completeness patterns for the schema of $D$. We say that $\mathcal{P}$ entails a pattern $(p, Q)($ or $\mathcal{P}$ entails $p$ for $Q)$ wrt. $D$, if all candidate completions in Cand $^{\mathcal{P}}(D)$ satisfy p for $Q$.

As discussed in Section 1 all patterns in Table 3 are entailed for $Q_{h w}$ by the base patterns in $D_{\text {maint }}$ wrt. $D_{\text {maint }}$.

It is easy to show that the problem to decide, given some $D, \mathcal{P}$, $Q$, and $p$, whether $\mathcal{P}$ entails $(p, Q)$ wrt. $D$, is coNP-complete in combined complexity and polynomial in data complexity (that is, fixing $(p, Q)$ and letting $D$ and $\mathcal{P}$ vary).

The problem that we investigate in the rest of this paper is, given a database $D$, base completeness patterns $\mathcal{P}$, and query $Q$, how to compute the set of all patterns $p$ entailed for $Q$, or a minimal representation of that set. 


\section{PATTERN ALGEBRA}

In Section 1 we have seen how query completeness patterns can be computed from base completeness patterns using algebra operations. In this section we formalize an algebra for completeness computations analogous to the SPJ fragment of relational algebra, which in turn captures the single-block SQL queries, and present experiments regarding the expected size of completeness patterns and pattern minimization techniques.

Unlike [17] and [22] which also operate at the schema level and do not take into account the database instance but are NP-complete for a fixed query with varying TC statements, the computations we describe below can be performed in PTIME. Also the pattern algebra presented here does not consider the database instance, but like in SQL, computations can be performed in PTIME for a fixed query. The algebra is intended to produce patterns that are correct and moreover as general as possible. Therefore, whenever possible, constants get replaced by wildcards. As we will show, the algebra produces always correct results, and is also complete for those conclusions that hold independently of the actual database instance.

\subsection{Algebra Definition}

The SPJ fragment of relational algebra with equality can be formalized using four operations: Selection by constant $\left(\sigma_{A=d}(R)\right)$, projection $\left(\pi_{\neg A}(R)\right)$, selection by attribute equality $\left(\sigma_{A=B}(R)\right)$ and the equijoin $\left(R_{1} \bowtie_{A=B} \quad R_{2}\right)$, where $R, R_{1}, R_{2}$ stand for generic data relations, not necessarily base relations, $A$ and $B$ for attributes, and $d$ for a constant.

Note that to ease the presentation, the projection $\pi_{\neg A}(R)$ used here is different from the classical projection. Instead of mentioning the surviving attributes, it mentions exactly one attribute that is projected out. For a given relation schema, classical projection can be modeled straightforwardly by this one, and vice versa.

In this section we define for each of the above operators an equivalent one operating on relations $P$ of completeness metadata. We make the distinction between data operators and metadata operators by adding a tilde character (e.g., $\tilde{\sigma})$ to the latter.

Decision support queries often include aggregation. Aggregation can be applied on top of the abovementioned operations. We discuss completeness calculation for aggregate queries in Appendix B

Next, we define each of the four operations of the pattern algebra. We suppose that $P$ is a relation with the same schema as $R$, but while $R$ contains data tuples, $P$ contains metadata, namely patterns describing the complete parts of $R$.

\subsubsection{Selection by Constant $\tilde{\sigma}_{A=d}(P)$}

For the selection $\sigma_{A=d}(R)$, intuitively, only those patterns in $P$ for $R$ give information about the output that have a wildcard or the value $d$ at position $A$. Other patterns are irrelevant.

Example 3. Consider the selection $\sigma_{\text {week }=2}$ (Warnings) in the introductory example. There are three completeness patterns for the table Warnings, namely $(*, 1, *, *)$, (Mon, $2, *, *)$ and (Wed, $2, *, *)$. For the result of the selection, the first pattern is irrelevant, as it talks about week 1 . The second and the third pattern are relevant, since they talk about week 2 . Furthermore, since the result may only contain records with week $=2$, we can generalize the last two patterns by replacing " 2 " with " $*$ ". Thus, the resulting metadata table looks as shown in Table 2

Let $p[A / *]$ denote the replacement of the value for attribute $A$ in $p$ with $*$. Then, for a metadata relation $P$, the selection for $\tilde{\sigma}_{A=d}(P)$ is defined as follows:

$$
\begin{aligned}
\tilde{\sigma}_{A=d}(P)= & \{p \in P \mid p[A]=*\} \\
& \cup\{p[A / *] \mid p[A]=d, p \in P\} .
\end{aligned}
$$

In summary, patterns with $A=*$ survive the selection unchanged, while patterns with $A=d$ survive but get generalized.

\subsubsection{Projection $\tilde{\pi}_{\neg A}(P)$}

Remember that we consider an atomic projection that projects away exactly one attribute.

Example 4. Consider the metadata part of the table Warnings, with the patterns $(*, 1, *, *)$, (Mon, $2, *, *)$ and (Wed, $2, *, *)$. If we project out the day attribute by $\pi_{\neg \text { day }}$ (Warnings), the projection $(1, *, *)$ of the first pattern still holds over the resulting table. For week 2, however, we cannot assert completeness, since e.g. records from Tuesday could be missing.

Generally, the projections of all completeness patterns with $A=*$ continue to hold over the projection of the data relation. Formally, the result of the projection $\tilde{\pi}_{\neg A}(P)$ over a metadata relation $P$ is defined as:

$$
\tilde{\pi}_{\neg A}(P)=\left\{\pi_{\neg A}(p) \mid p \in P, p[A]=*\right\} .
$$

\subsubsection{Selection by Attribute Equality $\tilde{\sigma}_{A=B}(P)$}

For a selection $\tilde{\sigma}_{A=B}$, those patterns say something about the result that subsume tuples with identical values for $A$ and $B$, that is, those patterns, that either have the same value for $A$ and $B$, or have a wildcard at at least one of the two positions.

Example 5. Suppose that $\left(d_{1}, d_{1}, e_{1}\right),\left(d_{2}, *, e_{2}\right)$ and $\left(*, *, e_{3}\right)$ are patterns for the table $R(A, B, C)$. Over the result of the selection $\sigma_{A=B}(R)$, all three completeness patterns continue to hold.

There are two particularities however: First, some generalized patterns also hold over the result, e.g. for the first pattern, we can replace one of the constants $d_{1}$ by a wildcard, yielding the two patterns $\left(d_{1}, *, e_{1}\right)$ and $\left(*, d_{1}, e_{1}\right)$ that are semantically equivalent over the data selection $\sigma_{A=B}(R)$.

Second, for patterns involving one wildcard and one constant, such as $\left(d_{2}, *, e_{2}\right)$, also the symmetric version holds, where the constant and the wildcard are swapped. In this case, this yields the pattern $\left(*, d_{2}, e_{2}\right)$. While the two patterns are semantically equivalent over the results of a selection, it is important that both be present, because possible subsequent projection operations that project out $A$ would lead to a loss of the first pattern, projections projecting out $B$ would lose the latter pattern.

For a pattern $p$, let $p[A \leftrightarrow B]$ denote the operation of swapping the values at positions $A$ and $B$. Then, the result of the operation $\tilde{\sigma}_{A=B}(P)$ is formally defined as follows:

$$
\begin{aligned}
\tilde{\sigma}_{A=B}(P)=\{p & \in P \mid p[A]=* \text { or } p[B]=*\} \\
& \cup\{p[A \leftrightarrow B] \mid p \in P, p[A]=* \text { or } p[B]=*\} \\
& \cup\{p[A / *] \mid p \in P, p[A]=p[B]\} \\
& \cup\{p[B / *] \mid p \in P, p[A]=p[B]\} .
\end{aligned}
$$

Example 6. Consider again the patterns $\left(d_{1}, d_{1}, e_{1}\right),\left(d_{2}, *, e_{2}\right)$ and $\left(*, *, e_{3}\right)$ from above. The result of the selection $\sigma_{A=B}(R)$ then satisfies exactly the patterns $\left(d_{1}, *, e_{1}\right),\left(*, d_{1}, e_{1}\right),\left(d_{2}, *, e_{2}\right)$, $\left(*, d_{2}, e_{2}\right)$ and $\left(*, *, e_{3}\right)$.

Note that in Table 3 for ease of presentation, we have omitted the generalizations and the symmetric tuples.

\subsubsection{Equijoin $P \tilde{凶}_{P . A=P^{\prime} . A^{\prime}} P^{\prime}$}

Completeness patterns behave in equijoins similar to records in regular equijoins, with the difference that the wildcard symbol $*$ joins with any constant. As in relational algebra, equijoins are equivalent to a combination of a cartesian product of the completeness patterns for $R$ and $R^{\prime}$ and a subsequent selection. 
Example 7. Suppose we want to compute the metadata join $P_{\text {Maint }} \tilde{凶}_{\text {resp=name }} \sigma_{\text {spec="hw" }}\left(P_{\text {Teams }}\right)$, where $P_{\text {Maint }}$ and $P_{\text {Teams }}$ denote the metadata of the tables Maintenance and Teams. The join corresponds to a subexpression of the algebraic expression in (1) that computes the query $Q_{h w}$. Then, we first compute a normal join between the patterns in the two tables, and afterwards introduce the symmetric versions for those patterns that involve one wildcard and one constant at the positions name and responsible. The input and output of this calculation are shown in Table 6

Formally, we define the result of a join $P \tilde{凶}_{P . A=P^{\prime} \cdot A^{\prime}} P^{\prime}$ as:

$$
\tilde{\sigma}_{P . A=P^{\prime} \cdot A^{\prime}}\left(P \times P^{\prime}\right) .
$$

Note that one need not actually compute a cartesian product, as the selection conditions can be pushed directly into the join, thus yielding a union of four smaller joins.

Aggregation. Decision support queries often contain aggregations such as COUNT, SUM, MIN or MAX. A discussion on how the pattern algebra can be extended to these aggregate functions is contained in Appendix B

Soundness and Completeness. In logic, an inference system is called sound, if all conclusions produced by the system are valid. An inference system is called complete, if the inference system produces all valid inferences.

We show below that the pattern algebra is a sound inference system, and that it is also complete, if the state of the database is ignored. To avoid confusion, we always write "computationally complete" when referring to the completeness of the algebra.

Intuitively, soundness means that whenever we have completeness patterns for an expression $E$ and apply one of the algebra operations to $E$, the resulting expression also satisfies the completeness patterns calculated by the analogous metadata operation.

Let $D$ be a database, $\mathcal{P}$ a set of base patterns for the schema of $D, Q$ a query and $p$ a pattern for the schema of $Q$. We write

$$
\mathcal{P}, D=(p, Q)
$$

to express that for every completion $D^{c} \in \operatorname{Cand}^{D}(\mathcal{P})$ it holds that $D^{c}$ satisfies $(p, Q)$ wrt. $D$. In other words, if $D^{c}$ is a completion of $D$ that satisfies the completeness assertions in $\mathcal{P}$ about the base tables of $D$, then the $p$-part of $Q(D)$ is the same as the $p$-part of $Q\left(D^{c}\right)$. The latter has been expressed formally in Def. 3 as $Q_{p}(D)=Q_{p}\left(D^{c}\right)$.

We write $\mathcal{P}, D \models(P, Q)$ in case $\mathcal{P}, D \models(p, Q)$ for every $p \in$ $P$. For queries in relational algebra analogous definitions apply.

Proposition 5 (Soundness). Let $D$ be a database, $\mathcal{P}$ be a collection of base patterns for the schema of $D$, and let $P$ and $P^{\prime}$ be sets of completeness patterns for algebra expressions $E$ and $E^{\prime}$, respectively. Furthermore, let op be an operation among $\left\{\pi_{\neg A}, \sigma_{A=d}, \sigma_{A=A^{\prime}}, \bowtie_{A=A^{\prime}}\right\}$. Then:

$$
\begin{aligned}
& \text { 1. } \mathcal{P}, D \models(P, E) \quad \text { implies } \mathcal{P}, D \models(\tilde{o p}(P), o p(E)) . \\
& \text { 2. } \mathcal{P}, D \models(P, E) \wedge\left(P^{\prime}, E^{\prime}\right) \quad \text { implies } \\
& \mathcal{P}, D \models\left(P \tilde{凶}_{A=A^{\prime}} P^{\prime}, E \bowtie_{A=A^{\prime}} E^{\prime}\right) .
\end{aligned}
$$

Proof. See Appendix A

Intuitively, the proposition says that if the patterns in $P$ are valid for $E(D)$, then those computed by $\tilde{o p}(P)$ are valid for $o p(E(D))$. Since the base patterns are valid for the base relations, we can conclude soundness for arbitrary expressions by induction.

Before proving the completeness of the pattern algebra, we have to differentiate patterns into meaningful and meaningless patterns.
Example 8. Consider the selection $\sigma_{\mathrm{spec}=}$ "hw" over the table Teams(name, specialization). Over the result of this selection, the pattern $(*$, software) can never subsume a record, as there can never be software teams in a table that was selected as only containing hardware teams.

We say that a query completeness pattern $(p, E)$ is satisfiable if there exists a database $D$ such that $E(D)$ contains at least one record that is subsumed by $p$.

Clearly, the pattern algebra cannot compute all entailed patterns, since there may be infinitely many. Instead, it will already be enough if it computes all satisfiable ones, and among those, only the maximally general ones. To make this formal, we need some notation.

Given a set of base completeness patterns $\mathcal{P}$, and a query pattern $(p, E)$, we write

$$
\mathcal{P} \models(p, E)
$$

if for all database instances $D$ it holds that $\mathcal{P}, D=(p, E)$.

If $E$ is an SPJ-algebra expression, then $\tilde{E}$ is the corresponding expression in the pattern algebra, obtained by replacing every operator in $E$ by its ${ }^{\sim}$-counterpart. Then $\tilde{E}(\mathcal{P})$ is the set of patterns produced by evaluating $\tilde{E}$ over $\mathcal{P}$.

Given a pattern $p$ and a pattern relation $P$, we write $p \preceq P$ if there is some pattern in $P$ that subsumes $p$.

We can now prove algorithmic completeness of the pattern algebra for satisfiable patterns when neglecting the state of the database.

Proposition 6 (COMPLETENESS Without InstanCE). Let $\mathcal{P}$ be a set of base completeness patterns, E an SPJ-algebra expression and $(p, E)$ a satisfiable query completeness pattern. Then

$$
\mathcal{P}=(p, E) \quad \text { implies } \quad p \preceq \tilde{E}(\mathcal{P}) .
$$

Proof. See Appendix A

The proposition states that if a satisfiable query completeness pattern $(p, E)$ is a logical consequence of $\mathcal{P}$, then we can evaluate the corresponding pattern algebra expression $\tilde{E}$ over $\mathcal{P}$ and find a $p^{\prime}$ in the result that is at least as general as $p$.

A corollary of soundness and completeness is that for any two equivalent relational algebra expressions, completeness patterns computed by the pattern algebra are equivalent.

One can show as another property of the pattern algebra that it never introduces redundancies, that is, if the completeness patterns for the base are minimal, then also the pattern sets computed by any algebra expression are minimal.

This means that for a an expression $E$ and a minimal $\mathcal{P}$ as input, the output $\tilde{E}(\mathcal{P})$ is identical for all equivalent expressions and thus does not depend on the specific expression.

\subsection{Experiments Using Wikipedia Data}

In all experiments that follow, the implementations were done in Java, using a PostgreSQL database, and executed on a machine with $2.4 \mathrm{Ghz}$ and 4 GB RAM.

To show the feasibility of our approach, we used a semiautomated scraping technique to identify 21 completeness statements from Wikipedia for tables about cities, countries and schools. We also extracted 10k schools and 200 countries from DBpedia, and $55 \mathrm{k}$ cities from OpenGeoDB and geodatasource.com. Note that DBpedia does not extract all the information in Wikipedia.

We then created seven join-queries over these tables and compared the time needed for query evaluation with the time needed for completeness calculation. The median query execution time was $2290 \mathrm{~ms}$, while the median completeness calculation time was 


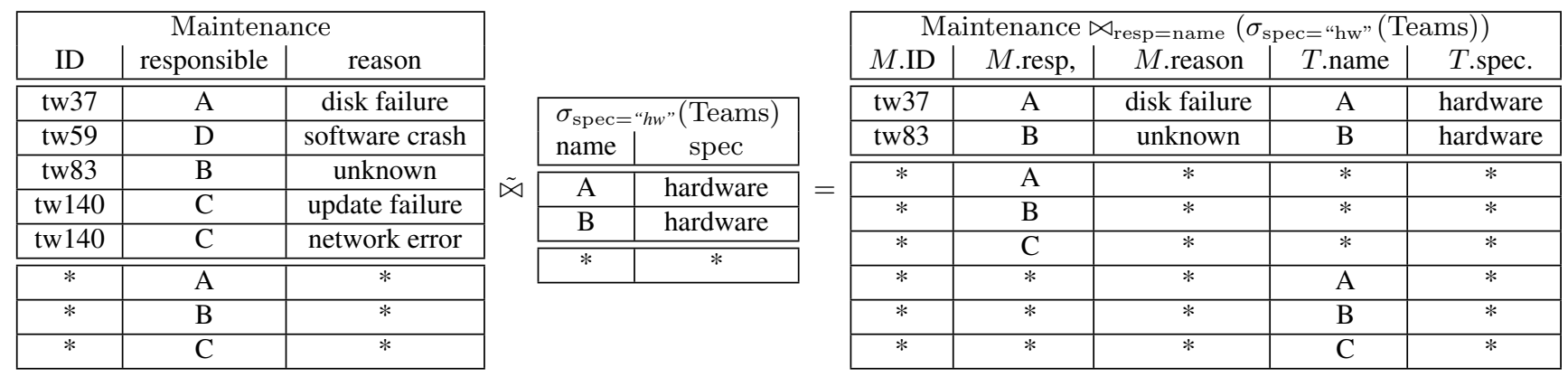

Table 6: Completeness information for the join between Maintenance and $\sigma_{\mathrm{spec}=\text { "hw" (Teams) }}$

$543 \mathrm{~ms}$, which implies that the completeness calculation took $23 \%$ of the time of the query calculation.

The query for which completeness calculation took longest compared with query evaluation was the query:

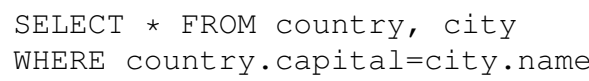

For this query, execution took $30 \mathrm{~ms}$ while completeness calculation took $797 \mathrm{~ms}$. The query for which the completeness calculation took shortest compared with the query evaluation was the query:

SELECT * FROM city, school

WHERE city.state $=$ school.state

For this query, execution took 175 seconds while completeness calculation took $421 \mathrm{~ms}$.

Both results can be explained by the size of the query results. While the first query is highly selective and produces only 278 records, the second produces 3 million records. In contrast, the number of completeness patterns computed for query results was between 9 and 100 (median 46) for the seven queries, and the time needed for their calculation exhibited a much lower variance with a range between 397 and $991 \mathrm{~ms}$.

The results for each query are listed in Table 7 in the Appendix.

\subsection{Number of Patterns}

To understand the possible overhead of computing query completeness patterns, it is necessary to understand how large sets of completeness patterns for base tables may actually be in applications. We synthetically created completeness patterns to measure the number of patterns wrt. the size of the data. The main conclusion is that over real data, the number of completeness patterns remains reasonably small compared with the normal data.

Test Case Generation. We believe that a typical situation may be one where most parts of a dataset are complete, and only a few records are missing. We also assume that in completeness patterns only attributes with a relatively low number of distinct values are used, which naturally correspond to the dimension tables in data warehouses.

After identifying such a set of attributes, we proceeded in our experiments as follows:

- We loaded a dataset.

- We assumed the most general pattern $(*, *, \ldots, *)$ to hold over the loaded dataset.

- We then repeatedly selected one record from the data set, which we dropped. Whenever some of the completeness patterns asserted for the dataset would subsume this record, they would cease to be true, so we dropped those patterns, and tried to add instead all possible most general specializations that continued to hold over the dataset.

For instance, if from the original Teams table we dropped a hardware team, the $(*, *, \ldots, *)$ pattern would not be correct any more. Instead, we would insert specializations that only assert completeness for software and networking teams.

We also experimented with two strategies for dropping records. In the first, the dropped records were selected purely at random. However, in reality, causes for missing data may lead to systematic data loss. We therefore investigated whether dropping related records leads to a different structure of completeness patterns than dropping random records.

Sample Cases. We first did experiments on a network element table from a network provider. The table has 64 attributes and 760k records. Among those 64 attributes, we manually identified six attributes that qualified as dimension attributes and seemed plausible for completeness patterns: region_name (6), technology (3), vendor (7), technology_capability_type (6), sector (13), and state (53), where the numbers in parentheses report the number of distinct values present in the data. Multiplying these numbers, we find a total of $1,185,408$ possible different completeness patterns. In the data, only 1,558 combinations $(0.205 \%)$ were present. Also, the frequency of these combinations was exponentially distributed.

As comparison we used the lineitem table from the TPC-H benchmark (scale factor 1), where we manually selected seven attributes which had few distinct values, leading to 460,800 possible completeness patterns. Of these, in the dataset of 6 million records, 73,419 combinations $(1.22 \%)$ were present.

Skewed data generators for TPC-H appear in the literature [5 8], but the tools are not publicly available. Also the more recent TPC-DS benchmark does not provide realistic data, as skew appears there only in a few fields such as "names", and fields are not correlated.

Experiments. We first dropped random records from our network element table. It turned out, that the number of completeness patterns converged after 300 dropped records at around 1000 completeness patterns. The likely explanation is that due to the few combinations in the real data set and due to the correlations, most dropped records have the same values as previously dropped records and therefore do not lead to further specialization of patterns. A graph is shown in Fig. 1

We tried the same method on the TPC-H dataset, where the number of completeness patterns did not show any convergence behaviour (same figure). A likely reason is that there are no correlations in the TPC-H dataset. 


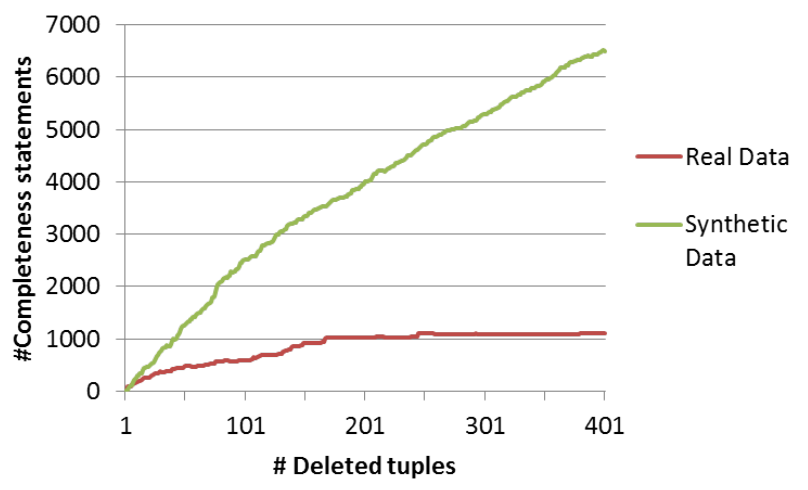

Figure 1: Growth of the number of completeness patterns for real data versus synthetic data.

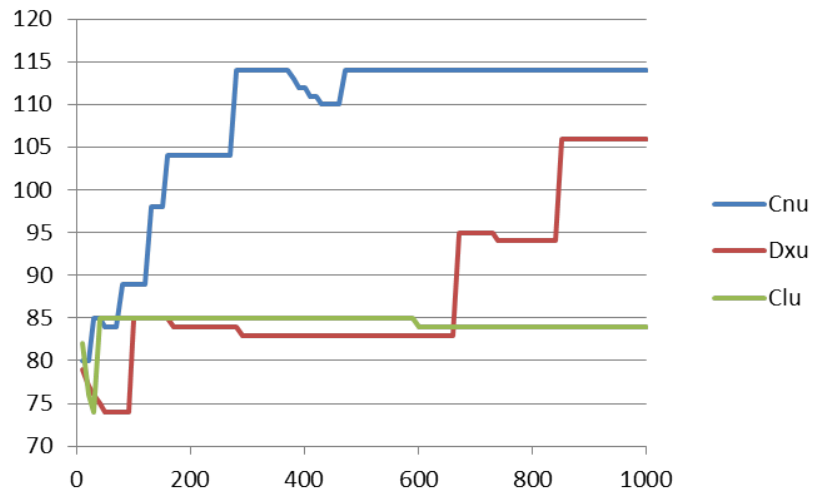

Figure 2: Number of completeness patterns for real data with systematic data loss.

Finally, we tested dropping related records. As indicator for relatedness we used the names of the network elements, because these names are not purely random but often carry some semantics. We dropped elements sharing three different prefixes of their names (Cnu, Dxu, Clu). It turned out that in all cases, the number of completeness patterns needed to describe the remaining complete data converged more quickly and was smaller. The results are shown in Fig. 2 Note that curves increase whenever some violated pattern can be specialized, and decrease if violated patterns cannot be specialized any more.

Conclusions. We draw three conclusions from these experiments: First, we see that the number of completeness patterns is relatively small compared to tuples in the data. Second, we see that when data loss is due to systematic reasons, the number of completeness patterns may be even smaller. Third, we see that this only applies to data sets which show skew and correlation, while for data sets that do not have these characteristics, the number of completeness patterns may be much higher.

\subsection{Minimization of Sets of Completeness Patterns}

Completeness patterns can subsume each other. Redundancies or replications could be reasons why that happens in practice, e.g. when first team A reports that data for itself is complete, and later a department head reports that data for all teams is complete. Redundancies can also occur due to additional inferences drawn when considering the state of the database instance, as will be shown in Section 5

A naive approach to minimization of sets of completeness patterns is pairwise comparison, however, already for 10k patterns, the runtime of this approach was more than 100 seconds, and the complexity is quadratic.

Since the experiments in the previous section showed that 1000 completeness patterns is a realistic input size, and pattern sets can grow quadratically in joins, we consider the minimization of sets of patterns a crucial operation.

There are two aspects to minimization that allow for optimization:

1. The approach how patterns are processed. We investigated three approaches, which we call all-at-once, incremental, and sorted incremental.

2. The choice of the data structure. We analyzed naive sets, hash tables, path indexes and discrimination trees, that latter two being data structures borrowed from the area of theorem proving, where a related problem of pattern minimization occurs.

We discuss each of these in detail below. As approaches and data structures are orthogonal, we use numbers 1 to 3 for referring to the approaches, and letters A to D for referring to the data structures.

Approaches. The first and most straightforward approach to minimization is (1) all-at-once processing. In this method, all patterns are loaded into memory, then for each pattern it is checked whether it is subsumed by some other pattern (see Subsection 3.2. Naively, this requires quadratic time, but data structures like hash tables, path indexes or discrimination trees can speed up this check.

Another approach is (2) incremental processing. Here, each pattern is loaded separately, and subsequently compared with the maximal patterns among the ones that already have been loaded. The comparison requires both to check whether the pattern is subsumed by one of the already loaded patterns (subsumption checking), and, if that is not the case, to find the patterns among the already loaded ones that are subsumed by the new pattern (supersumption retrieval).

An extension of incremental processing is (3) sorted incremental processing. When completeness patterns are sorted in decreasing order wrt. the number of wildcards they contain, then no later pattern can entail an earlier pattern, because subsumption requires that the subsuming pattern has more wildcards than the subsumed pattern (except for duplicate patterns). Therefore, incremental sorted processing does not require supersumption retrieval.

The advantages of the incremental approaches are that, given that the set of maximal patterns is sufficiently smaller than the input, these methods require less memory.

Data Structures. Lists (A) are the most basic data structure, which we use as baseline. Using lists, a single subsumption check or supersumption retrieval requires linear time wrt. the number of patterns, thus leading to a quadratic algorithm.

Hash tables $(B)$ can be used to achieve faster subsumption checks: Given a pattern, checking whether it is subsumed by some stored pattern can be done by explicitly generating all generalizations of that pattern, and checking whether any of those is stored in the hash table. However, the number of generalizations of a pattern is exponential in the number of constants in the pattern, as each way of replacing some constants with wildcards yields a generalization. 


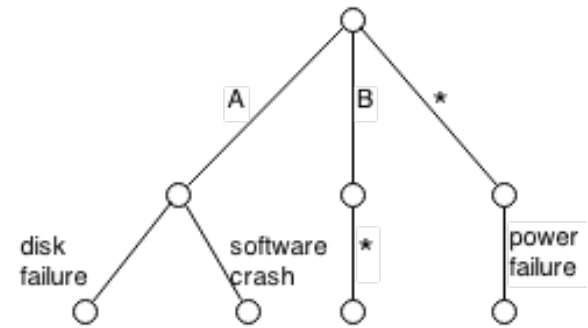

Figure 3: A discrimination tree over two attributes (team,message), storing the patterns (A, disk failure), (A, software crash), $(\mathrm{B}, *)$ and $(*$,power failure).

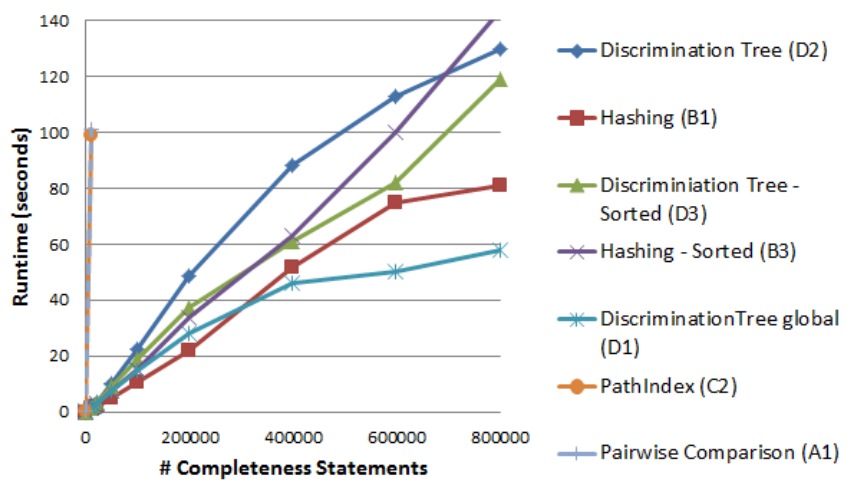

Figure 4: Runtime comparison of pattern minimization techniques.

In the AI community, similar problems occur when managing sets of terms used in theorem provers. Besides the subsumption checks and supersumption retrieval, theorem provers also need to be able to find instantiations and unifiable patterns. Also, theorem provers need to deal with terms that involve repeated variables and function symbols. There are two classical techniques for term indexing: discrimination trees and path indexing [20].

Path indexes $(C)$ are similar to inverted indexes. The idea is to create a list of occurrences for each symbol (constants and wildcard) at each position. To find subsumed and supersumed patterns, relevant lists then have to be intersected or merged. Especially the intersection is expensive, leading to a poor performance of the method in our experiments.

Discrimination Trees $(D)$ are essentially tries over completeness patterns, which treat the wildcard value in the same way as other constants. An example of a discrimination tree is shown in Fig. 3 Given a pattern $p$, subsumption checking is done by a search from the root, which at any node $n$ at level $i$ always continues searching in branches labelled with $*$, and if $p[i]=d$, also searches branches labelled with $d$. Thus, subsumption checking requires search with a branching factor of at most 2. Supersumption retrieval, on the other hand, considers the branch labelled with $d$, if $p[i]=d$, and if $p[i]=*$, then all branches. Thus, the branching factor is only limited by the branching factor of the discrimination tree.

Experiments. In the experiments, we used random subsets of one million completeness patterns generated as the result of a cartesian product between two tables with 1000 patterns each.

In Fig. 4, we show the runtime comparison. It turned out that the all-at-once approach was faster than the two other approaches, and that for this approach, discrimination trees (D1) were $25 \%$ faster

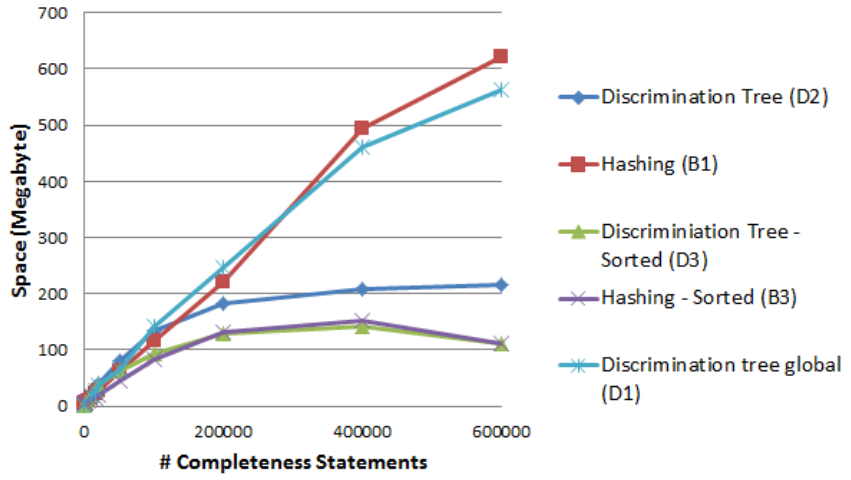

Figure 5: Space comparison of minimization using discrimination trees and hashing.

than hashing (B1). Pairwise comparison (A1) and path indexes (C2) turned out to be totally inapplicable, the latter possibly due to the few attribute values appearing in our use case.

In Fig. 5. we compare different approaches in combination with discrimination trees and hash tables regarding the space needed. Here, the sorting approaches (B3 and D3) perform clearly best while the all-at-once approaches, as expected, consume an amount of space that is linear in the input size. The fact that the sorted approaches consume even less space for $600 \mathrm{k}$ patterns than for $400 \mathrm{k}$ is consistent with calculations that show that the number of maximal patterns decreases when using more than $300 \mathrm{k}$ random patterns out of the one million patterns.

Conclusions. We conclude that regarding space, approaches that sort the input first perform best, while regarding runtime, approaches that process all patterns at once perform best. While regarding space there is no difference between the best discrimination tree and the best hashing method, regarding runtime, discrimination trees are consistently faster than hashing. The advantage of discrimination trees is likely to increase when more attributes (here twelve) are used for completeness patterns.

\section{MAKING THE PATTERN ALGEBRA INSTANCE-AWARE}

So far we have computed completeness patterns for queries only from the patterns that hold for base relations. However, as seen in the motivating example, additional inferences are possible when taking into account also the tuples in the database instance. In this section we argue that such an extension of the pattern algebra brings about benefits, show that instance awareness may be expensive in theory, and evaluate the efficiency of the extended algebra on a test case.

\subsection{Join Extension}

In the motivating example we saw that patterns in a join result can sometimes be summarized to more general patterns. Let us look at that case again in more detail.

Example 9. Consider again the join

$$
M \bowtie_{M . \text { resp=T.name }} \sigma_{\mathrm{spec}=" \mathrm{hw}}(T),
$$

whose computation is shown in Table 6 We see that the only completeness pattern for $\sigma_{\mathrm{spec}=}$ "hw" $(T)$ is $(*, *)$. We also see that the only records in $T$ that match this pattern are (A, hardware) and 
( $\mathrm{B}$, hardware). This allows us to conclude that all records appearing in the join result must have either the value A or B for the attributes $M$.resp and $T$.name. We also see that the metadata for $M$ contains two patterns $(*, \mathrm{~A}, *)$ and $(*, \mathrm{~B}, *)$, which contain exactly the values $\mathrm{A}, \mathrm{B}$ in the second position, the join position, and which are identical in the other positions.

We therefore conclude that these two patterns cover all possible values that can appear in the join result, and that the result patterns $(*, \mathrm{~A}, *, *, *)$ and $(*, \mathrm{~B}, *, *, *)$ can therefore be promoted to the more general pattern $(*, *, *, *, *)$ for the join result. As a consequence, we know that the entire results of the join is complete.

The preceding example illustrates that promotion of statements gives some benefits. First, it allows one to identify larger parts of the query result as complete. Moreover, the new pattern subsumes all the promoted patterns and thus leads to a more compact representation. More general statements with a $*$ in more attribute positions can also survive more projection operations, since a pattern $p$ over $R$ only gives rise to an element in $\pi_{\neg A}(R)$ if $p[A]=*$. As discussed in Appendix $B$ aggregation bears some similarity with projection and thus more general statements allow one also to recognize more results of an aggregate query as correct.

Next, we show with an extended example how based on this idea one can define a general promotion technique for joins. Consider two tables $R(A, B, C), R^{\prime}\left(A^{\prime}, B^{\prime}\right)$ as shown below, and the join query $R \bowtie_{A=A^{\prime}} R^{\prime}$.
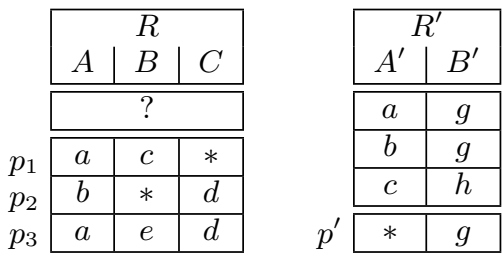

Clearly, all three patterns of $R$ join with the pattern of $R^{\prime}$, because the latter has a " $*$ " in the join position. The result records of the join all have the constant $a$ or $b$ in the join positions because $R$ does so. We show how we can infer more general completeness patterns if we take into account the records in $R^{\prime}$.

Let us consider first the pattern $p^{\prime}$ over $R^{\prime}$. The only records in $R^{\prime}$ that match $p^{\prime}$ are $(a, g)$ and $(b, g)$, and since $p^{\prime}$ is a completeness pattern, these are all such records that one will ever find in $R^{\prime}$. Thus, the only values that can possibly occur for attribute $A^{\prime}$ in a match of $p^{\prime}$ in $R^{\prime}$, the "allowable domain" for $A^{\prime}$ with respect to $p^{\prime}$, so to speak, are $a$ and $b$. We can retrieve these values from the instance $D$ with the project-select query $\pi_{A^{\prime}}\left(\sigma_{B^{\prime}=g}\left(R^{\prime}(D)\right)\right)$ where $R^{\prime}(D)$ denotes the instance of $R^{\prime}$ in $D$.

Now, we move to the patterns for $R$. Suppose, the pattern $p_{4}=$ $(*, c, d)$ would hold over $R$. Then we could conclude that the concatenation $p_{4} \cdot p^{\prime}=(*, c, d, *, g)$ holds over the join $R \bowtie_{A=A^{\prime}} R^{\prime}$. In fact, $p_{4} \cdot p^{\prime}$ does hold over the join. Since $p_{1}$ holds over $R$, also the specialization $(a, c, d)$ holds, and since $p_{2}$ holds over $R$, also the specialization $(b, c, d)$ holds. Thus, both $(a, c, d, *, g)$ and $(b, c, d, *, g)$ hold over the join. However, as seen above, $a$ and $b$ are the only possible values for $A^{\prime}$ in $R^{\prime}$, and thus, they are also the only possible values for $A$ in the join $R \bowtie_{A=A^{\prime}} R^{\prime}$. Consequently, $(*, c, d, *, g)$ holds, which has the "*" in $A$ that, intuitively, has been transferred from $A^{\prime}$. We say that $(*, c, d, *, g)$ has been obtained by "promoting" the statements $p_{1}$ and $p_{2}$ using $p^{\prime}$.

Allowable Domain: Let us discuss which difficulties can arise in applying this technique. We make a promotion attempt for every pattern $p^{\prime}$ over $R^{\prime}$ that has a "*" in the join position $A^{\prime}$. To this end, we construct a project-select query out of $p^{\prime}$ that retrieves the allowable domain $\Delta_{A^{\prime}}^{p^{\prime}}$ for $A^{\prime}$ with respect to $p^{\prime}$, which consists of all $A^{\prime}$-values of records in $R^{\prime}$ that match $p^{\prime}$. In the example above, we had $\Delta_{A^{\prime}}^{p^{\prime}}=\{a, b\}$. This step is easy.

Choice Sets: Having identified the allowable domain, we look for all possible choice sets of patterns over $R$, that is, sets that contain for each value in $\Delta_{A^{\prime}}^{p^{\prime}}$ one statement with that value for the join attribute $A$. In the example above, $\{(a, c, *),(b, *, d)\}$ was a choice set, and $\{(a, e, d),(b, *, d)\}$ is another one. A potential difficulty is that there may be exponentially many choice sets.

Then we check for every choice set whether, together with the initial pattern $p^{\prime}$ over $R^{\prime}$, it can be promoted. To this end, we replace the $A$-values in the records of the choice set with a " $*$ " and check whether the resulting pattern set is unifiable, that is, whether for every attribute position there is at most one constant that occurs in that position in the set. In the example above we obtain $\{(*, c, *),(*, *, d)\}$, and $\{(*, e, d),(*, *, d)\}$, which clearly are unifiable.

Unification: If the check is positive, we compute the unifier, that is, the most general pattern that matches every pattern in the set. The unifier has a constant in those positions for which there is a (single) constant in the set and has a " $*$ " in the other ones. Thus, the unifiers in our example are $(*, c, d)$ and $(*, e, d)$. Checking unifiability and computing unifiers is conceptually easy.

The promoted statements are then obtained by concatenating the unifiers of the unifiable choice sets with the initial pattern $p^{\prime}$. Thus, in the example, the promoted statements are $(*, c, d, *, g)$ and $(*, e, d, *, g)$.

So far, we have discussed how to use statements over $R^{\prime}$ to promote statements over $R$. Of course, this can also be done in the reverse direction, from $R$ to $R^{\prime}$.

To incorparate promotion into the pattern algebra, we introduce the join operator

$$
P \hat{凶}_{A=A^{\prime}} P^{\prime}
$$

that first computes $P \tilde{凶}_{A=A^{\prime}} P^{\prime}$ and then adds all patterns that can be obtained by promotion in either direction. The algebra with this join operator is the instance aware algebra.

Soundness and Completeness. As for the pattern algebra, the computational properties of the extended algebra are of interest.

It is easy to show that the instance-aware algebra is sound. Regarding the computational completeness, we conjecture that the algebra is complete for inferences wrt the state of the database, for all queries in which no attribute is reused in joins (e.g. the query $Q_{h w}$ is in this class). As we show however in Appendix E for general queries the pattern algebra with promotion is still not complete.

\subsection{Runtime and Space Effects of Promotion}

Promotion is possibly expensive, because for an initial pattern $p^{\prime}$ over $R^{\prime}$ we iterate over all choice sets of patterns over $R$, to perform the unifiability check and possibly compute a promoted statement. As the number of subsets of a set and therefore the number of choice sets can be exponential, this iteration may be expensive. Furthermore, as each unifiable choice set gives rise to a new completeness pattern, the output of promotion may also have exponential size.

We discuss here possible optimizations, and present experimental results regarding the runtime and space effects of promotion.

Implementation of Promotion. Given an initial pattern $p^{\prime}$ over $R^{\prime}$, promotion considers all choice sets of patterns over $R$ that contain one pattern $p$ with $p[A]=d$ for every $d \in \Delta_{A^{\prime}}^{p^{\prime}}$. It 
is natural to implement the procedure to find all such choice sets $S$ by first splitting $P$, the set of patterns over $R$, into so-called $A$ sets, that is, one set for each value appearing at position $A$. Given $\Delta_{A^{\prime}}^{p^{\prime}}$, choice sets $S$ to test for unification are then all sets which are composed by choosing exactly one pattern from each $A$-set whose value appears in $\Delta_{A^{\prime}}^{p^{\prime}}$

There exist a few optimizations to this procedure:

- Trivial failure: If one $A$-set required by $\Delta_{A^{\prime}}^{p^{\prime}}$ is empty, promotion is impossible.

- Pruning: Instead of choosing one pattern from each $A$-set and then testing for unification, one can test unification "onthe-go", whenever choosing one more pattern. Thus, if e.g. $\Delta_{A^{\prime}}^{p^{\prime}}$ has size 5 but already the first two chosen patterns are not unifiable, there is no need to choose patterns from the other $A$-sets.

- Subsumption detection: Similarly to pruning, if an intermediate unifier is already more specific than a pattern computed for the result, any patterns retrieved using this unifier will be redundant and therefore the search can be pruned.

- Due to the previous optimizations, the iteration order of the $A$-sets can play a role. As common in search problems, it turned out that starting with the smallest sets gave the best results.

In the following experiments, these optimizations reduced the number of sets to be tested by $40 \%$ to $99 \%$.

Experiments. When using pattern promotion, two questions arise: first, how the runtime is affected by iterating through possibly exponentially many sets, and second, what effect promotion has on the number of patterns in the output of a join.

We evaluated both aspects in two typical scenarios. The first is a join of a fact table with a dimension table, which is considered to be complete. The second is a join between two partially complete fact tables. For both experiments, we used the network element table from Section 4.3 , and in the latter case performed a selfjoin.

The results for the join of the fact table with the dimension table were that the median runtime of the join was between 91 and 661 milliseconds for various join attributes, opposed to a table scan taking 37 seconds. Nevertheless, for the two attributes with the highest cardinality, $5 \%$ and $10 \%$ of the runs took longer than 30 seconds and were considered as timeouts.

For the join of the two partially complete fact tables, we observed a quadratic growth of the runtime wrt. the number of input patterns, similarly as one would expect it from normal joins.

For both kinds of join, the promoted patterns, instead of leading to a bigger output, lead to a smaller output, because they subsumed other statments in the output. For the join of the two partially complete tables, this reduction was especially significant, causing a reduction between $80 \%$ and $95 \%$. In no case promotion lead to an increased output size. More details on the experiments and graphs are shown in Appendix D

\section{DISCUSSION}

In this section we discuss aspects concerning the source of completeness patterns and implementational issues.

Source of Completeness Patterns. We have already discussed the source of completeness information in some scenarios. To generalize those observations, we believe that in domains where data is loaded automatically, such as stream processing [12] or feed management [25], completeness patterns should also be generated in an automated way by the loading system. On the other hand, in applications where data is created by humans, completeness patterns cannot be automatically generated. Instead, their creation should become part of the workflow used to enter the data. Concretely, web interfaces that allow to enter data could e.g. contain fields that allow to specify that all data of a certain kind was entered.

Storage. A first question is how to store completeness patterns in an RDBMS. The conceptually straightforward solution is to introduce one metadata table for each table. For storing the wildcard value ("*"), one either has to use new data types that extend previous data types by allowing the wildcard value, or, for data types such as string, one may use string escaping techniques.

Placement of Reasoner. The next question is the placement of the completeness reasoner. It could either be placed within the DBMS itself, e.g. as plugin, or it could be placed outside as an independent component. While the pattern algebra works only on the schema level, the subsequently introduced promotion operator needs access to the database content, and would therefore benefit from fast access to the database content. The advantage of placing the reasoner outside the DBMS would be that the reasoner could work independently from a specific database.

Plan Generation and Execution. One way to do completeness reasoning is to attach it to the normal query evaluation: While the query result is computed in the database, for each algebraic operation applied to the data, in parallel, we execute the corresponding operation on the metadata. This may however lead to sub-optimal computation of completeness. Because the metadata can be very different from the normal data in size and distribution, the optimal plan for query computation may not be the optimal plan for completeness calculation. A proper implementation might therefore benefit from having its own cost model to use for optimizing the metadata query plan. We leave this as future work.

\section{CONCLUSION}

In this paper we have discussed that in many applications such as loosely coupled cloud databases, collaborative editing and network monitoring, databases should be considered partly under closedworld and partly under open-world semantics, and showed how that can be formalized using completeness patterns. We then investigated the problem of computing completeness patterns for query results over such databases. We presented the pattern algebra, which operates purely at the schema level and can be executed analogously to relational algebra, and then discussed extensions using the promotion operator to make use of inferences possible due to the database instance. We highlighted implementational challenges regarding the minimization of sets of completeness patterns and the computation of promoted completeness patterns. We used two real data sets to empirically demonstrate the utility and scalability of our techniques in practice.

An interesting extension would be to take into account constraints such as keys, foreign keys, inclusion or functional dependencies.

\section{Acknowledgements}

We are thankful to Stephan Schulz for advice concerning relevant index structures. We are also thankful to the reviewers of this paper for pointing us to the work by Lang et al. [16]. This work has been partially supported by the project "MAGIC: Managing Completeness of Data" funded by the province of Bozen-Bolzano, Italy. 


\section{REFERENCES}

[1] S. Abiteboul, P. Kanellakis, and G. Grahne. On the representation and querying of sets of possible worlds. In SIGMOD, pages 34-48, 1987.

[2] A. C. Acock. Working with missing values. Journal of Marriage and Family, 67(4):1012-1028, 2005.

[3] S. Auer, C. Bizer, G. Kobilarov, J. Lehmann, R. Cyganiak, and Z. Ives. Dbpedia: A nucleus for a web of open data. Springer, 2007.

[4] L. Berti-Equille, T. Dasu, and D. Srivastava. Discovery of complex glitch patterns: A novel approach to quantitative data cleaning. In ICDE, pages 733-744. IEEE, 2011.

[5] N. Bruno and S. Chaudhuri. Flexible database generators. In VLDB, pages 1097-1107, 2005.

[6] K. L. Clark. Negation as failure. In Logic and data bases, pages 293-322. Springer, 1978.

[7] A. Cortés-Calabuig, M. Denecker, O. Arieli, and M. Bruynooghe. Representation of partial knowledge and query answering in locally complete databases. In Logic for Programming, Artificial Intelligence, and Reasoning, pages 407-421, 2006.

[8] A. Crolotte and A. Ghazal. Introducing skew into the TPC-H benchmark. In TPCTC, pages 137-145, 2011.

[9] M. Denecker, A. Cortés-Calabuig, M. Bruynooghe, and O. Arieli. Towards a logical reconstruction of a theory for locally closed databases. ACM TODS, 35(3), 2010.

[10] W. Fan and F. Geerts. Relative information completeness. In PODS, pages 97-106, 2009.

[11] W. Fan and F. Geerts. Capturing missing tuples and missing values. In $P O D S$, pages 169-178, 2010.

[12] L. Golab and T. Johnson. Consistency in a stream warehouse. In CIDR, pages 114-122, 2011

[13] J. M. Hellerstein. Quantitative data cleaning for large databases. United Nations Economic Commission for Europe (UNECE), 2008.

[14] C. R. Kalmanek, Z. Ge, S. Lee, C. Lund, D. Pei, J. Seidel, J. Van der Merwe, and J. Yates. Darkstar: Using exploratory data mining to raise the bar on network reliability and performance. In $D R C N$, pages 1-10. IEEE, 2009.

[15] W. Lang, R. V. Nehme, and I. Rae. Database optimization for the cloud: Where costs, partial results, and consumer choice meet. In CIDR, 2015.

[16] W. Lang, R. V. Nehme, E. Robinson, and J. F. Naughton. Partial results in database systems. In SIGMOD, pages 1275-1286. ACM, 2014.

[17] A. Y. Levy. Obtaining complete answers from incomplete databases. In $V L D B$, pages 402-412, 1996.

[18] L. Libkin. Incomplete data: what went wrong, and how to fix it. In PODS, 2014, pages 1-13, 2014.

[19] D. Maier and P. A. Tucker. Punctuations. In Encyclopedia of Database Systems, pages 2216-2217. Springer, 2009.

[20] W. McCune. Experiments with discrimination-tree indexing and path indexing for term retrieval. Journal of Automated Reasoning, 9(2):147-167, 1992.

[21] A. Motro. Integrity = Validity + Completeness. ACM TODS, 14(4):480-502, 1989.

[22] S. Razniewski and W. Nutt. Completeness of queries over incomplete databases. In $V L D B, 2011$

[23] R. Reiter. On closed world data bases. Springer, 1978.

[24] P. Royston. Multiple imputation of missing values. Stata Journal, 4:227-241, 2004.

[25] V. Shkapenyuk, T. Johnson, and D. Srivastava. Bistro data feed management system. In SIGMOD, pages 1059-1070. ACM, 2011.

[26] M. Yakout, L. Berti-Équille, and A. K. Elmagarmid. Don't be scared: use scalable automatic repairing with maximal likelihood and bounded changes. In SIGMOD, pages 553-564. ACM, 2013.

\section{APPENDIX}

\section{A. PATTERN ALGEBRA PROPERTIES}

In this section we give the proof for the soundness of the pattern algebra, and for the completeness of the pattern algebra when the state of the database instance is neglected.
Proposition 5 (Soundness). Let $D$ be a database, $\mathcal{P}$ be a collection of base patterns for the schema of $D$, and let $P, P_{1}$ and $P_{2}$ be sets of completeness patterns for algebra expressions $E, E_{1}$ and $E_{2}$, respectively. Furthermore, let op be an operation among $\left\{\pi_{\neg A}, \sigma_{A=d}, \sigma_{A=B}\right\}$. Then:

$$
\begin{aligned}
& \text { 1. } \mathcal{P}, D \models(P, E) \quad \text { implies } \mathcal{P}, D \models(\tilde{o p}(P), \text { op }(E)) . \\
& \text { 2. } \mathcal{P}, D \models\left(P_{1}, E_{1}\right) \wedge\left(P_{2}, E_{2}\right) \quad \text { implies } \\
& \mathcal{P}, D \models\left(P_{1} \tilde{凶}_{A=B} P_{2}, E_{1} \bowtie_{A=B} E_{2}\right) .
\end{aligned}
$$

Proof. 1. $o p=\sigma_{A=d}$ (Selection by constant): This operation is correct for two reasons: (1) because patterns with $A=*$ clearly continue to hold when some tuples are dropped; (2) because the result of a query $\sigma_{A=d}(E)$ cannot contain any tuples $t$ with values $t[A] \neq d$, and therefore, whenever a pattern $p$ with $p[A]=d$ is in the metadata, the same pattern can also be generalized by replacing $d$ at position $A$ with $*$.

1. op $=\pi_{\neg A}$ (Projection): This operation is correct because wildcards cover all possible values and may therefore be projected out.

1. $o p=\sigma_{A=B}$ (Selection by attribute equality): This operation is correct, because the query result table can only contain tuples $t$ with $t[A]=t[B]$, and thus, completeness patterns $(d, d, \ldots)$, $(d, *, \ldots)$ and $(*, d, \ldots)$ are equivalent (assuming wlog that $A, B$ are the first two attributes).

2. $\bowtie$ (Equijoin): This operation is correct, because, as for selection by attribute equality, the result can only contain tuples satisfying symmetry, and thus, the various patterns $(d, d, \ldots),(d, *, \ldots)$ and $(*, d, \ldots)$ produced by the variable selection on top of the cartesian are equivalent.

Proposition 6 (COMPleteness Without Instance). Let $\mathcal{P}$ be a set of base completeness patterns, E an SPJ-algebra expression and $(p, E)$ a satisfiable query completeness pattern. Then

$$
\mathcal{P} \mid=(p, E) \quad \text { implies } \quad p \preceq \tilde{E}(\mathcal{P}) .
$$

Proof. We prove the claim by induction over the height of the expression $E$.

Base case: The height of the expression $E$ is one, that is, the expression is exactly some base table $R$. Then, the completeness patterns for $E$ are exactly the completeness patterns of the form $(p, R)$ in $\mathcal{P}$, and thus the algebra is complete.

Induction hypothesis: The algebra is complete for expressions up to size $n$.

Induction step: Show completeness for expressions of size $n+1$.

We make a distinction of cases, depending on the outermost operator in $E$.

Selection by constant: Suppose $E$ is of the form $\sigma_{A=d}\left(E^{\prime}\right)$ and $(p, E)$ is satisfiable. Let $D$ be a database that satisfies $(p, E)$. Then there is a $t \in E(D)$ such that $p$ subsumes $t$. As $t[A]=d$, if follows that $p[A]$ is either $d$ or $*$.

Since $(p, E)$ follows from $\mathcal{P}$, this implies that in all completions $D^{c}$ for any $D$ wrt. $\mathcal{P}$, the equality $\sigma_{p=\operatorname{attr}(E)}(E(D))=$ $\sigma_{p=\operatorname{attr}(E)}\left(E\left(D^{c}\right)\right)$ holds.

We can now move the selection $\sigma_{A=d}$ from $E$ directly into the outward selection and find that also $\sigma_{p[A / d]=\operatorname{attr}\left(E^{\prime}\right)}\left(E^{\prime}(D)\right)=$ $\sigma_{p[A / d]=\operatorname{attr}\left(E^{\prime}\right)}\left(E^{\prime}\left(D^{c}\right)\right)$ holds. But this implies that $\mathcal{P}$ entails the completeness pattern $\left(p[A / d], E^{\prime}\right)$, and hence by the induction hypothesis it holds that $p[A / d] \preceq \tilde{E}^{\prime}(D)$.

By the defininition of the algebra operation for selection by constant, it follows that $p \preceq \tilde{E}(D)$.

Projection: Suppose $E$ is of the form $\pi_{\neg A}\left(E^{\prime}\right)$ and $(p, E)$ is satisfiable. 
It follows that in all completions $D^{c}$ for any $D$ wrt. $\mathcal{P}$, the equality $\sigma_{p=\operatorname{attr}(E)}(E(D))=\sigma_{p=\operatorname{attr}(E)}\left(E\left(D^{c}\right)\right)$ holds.

By the inclusion $D \subseteq D^{c}$, we can also include the attribute $A$ that was projected out into the condition of the selection, and arrive at the equality $\sigma_{(*, p)=\operatorname{attr}\left(E^{\prime}\right)}\left(E^{\prime}(D)\right)=\sigma_{(*, p)=\operatorname{attr}\left(E^{\prime}\right)}\left(E^{\prime}\left(D^{c}\right)\right)$ (because, due to the bag semantics and the inclusion, any violation of the latter equation implies also a violation of the former equation).

But this implies that $\mathcal{P}$ entails the query completeness pattern $\left((*, p), E^{\prime}\right)$ and hence, by the induction hypothesis, it holds that $(*, p) \preceq \tilde{E}^{\prime}(\mathcal{P})$. By the defininition of the algebra operation for projection, it follows that $p \preceq \tilde{E}(\mathcal{P})$.

The cases of selection by attribute equality and of equijoin are analogous.

\section{B. AGGREGATION}

Decision-support queries often include aggregation. For instance, it may be interesting to count the number of cities in a certain country, to sum up populations, or to obtain the country with the largest population.

The pattern algebra can naturally be extended to aggregation operators. Because aggregation corresponds to a form of negation, as Lang et al. observed [16], incompleteness of the database may lead to incorrect records in query answers.

Consider for instance a query for the number of cities in each country. As seen in Table 4 Wikipedia is complete for all cities in Bulgaria, but not for all cities in France. So if in reality there were 700 cities in France, but Wikipedia contains only 200, then the query answer will not only be incomplete (it misses the record (France, 700)), but also incorrect (it contains the record (France, 200).

Fortunately, completeness patterns as derived by the pattern algebra also guarantee correctness. Whenever cities in France are complete, this implies that the number of cities in France is also correct.

In our framework, one can extend the pattern algebra by additional operators for simple aggregation operations, that project away all attributes not mentioned in group-by statements.

For instance, the query

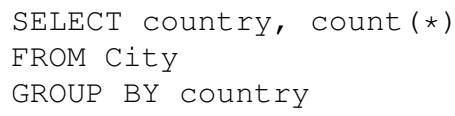

could be computed using a count operator on top of $\pi_{\text {country }}$ (City).

The count operator would extend completeness patterns by a wildcard for the count value: Given the statements in Table 4 the query for the number of cities per country would satisfy the following patterns: (Germany,*), (Ukraine,*), (Bulgaria,*). Extensions for the aggregate functions SUM, MIN and MAX are analogous.

\section{QUERY STATISTICS IN THE WIKIPEDIA USE CASE}

In Table 7 we report the result size and the compute time for the SQL queries used in the experiments in Section 4.2

\section{PATTERN PROMOTION EXPERIMENTS}

In the following, we give details about the evaluation of the runtime and the space growth due to promotion in use cases. We use the same network element table as in Section 4.3 . and focus on two kinds of joins:
The first and in a data warehouse most straightforward case is the join of a fact table with a dimension table. It is reasonable to assume that dimension tables are complete. We therefore model this case by joining the table from our use case, augmented with completeness patterns using the method presented in Section 4.3 , with a unary second table that contains a random subset of all values appearing for the join attribute in the use case table (these values determine $\mathrm{Val}$ in the algorithm), and where the second table is asserted to be complete.

The second case is the join between two fact tables, which are both only partially complete. We simulate this by a selfjoin between two versions of our use case table.

Join with a Dimension Table. The first experiment was on measuring the number of sets $S$ that had to be checked for unifiability by the promotion algorithm. As input we used 1000 completeness patterns. Table 8 shows the result of these experiments from 100 runs for each row. One can see that despite the large number of combinations $S$ to be tested naively, the calculations are feasible in most cases. However, for two attributes, namely state and sector, some runs exceeded a timeout threshold of 30 seconds. As we can also see, the median runtimes were between 91 and 661 milliseconds. For comparison, a table scan of the network element table with 700,000 rows took 37 seconds. We also see that the number of patterns in the output is smaller than the input, and that the number of promoted patterns is comparably small. This means that, by subsuming other patterns, the promoted patterns lead to a reduction of the output size.

The reason why attributes behave differently even if they have a similar number of distinct values is probably the distribution of these values. E.g. the attribute tech_capability has one value occurring with $>99.9 \%$ frequency.

We draw two conclusions: First, the runtime is feasible in most cases, though there may also be some timeouts, and second, promotion leads to a reduction of the number of patterns in the output.

\begin{tabular}{|c|c|c|c|c|c|}
\hline $\begin{array}{l}\text { Join } \\
\text { attribute }\end{array}$ & $\begin{array}{l}\text { Number } \\
\text { of distinct } \\
\text { attribute } \\
\text { values }\end{array}$ & $\begin{array}{l}\text { Size of } \\
\text { minimized } \\
\text { output } \\
\text { (avg) }\end{array}$ & $\begin{array}{l}\text { Promoted } \\
\text { statements } \\
\text { in minimi- } \\
\text { zed output }\end{array}$ & $\begin{array}{l}\text { Size of S } \\
\text { naïve } \\
\text { (avg) }\end{array}$ & $\begin{array}{l}\text { Runtime } \\
\text { in ms } \\
\text { (median) }\end{array}$ \\
\hline vendor & 7 & 515 & 45 & $1 * 10^{7}$ & 270 \\
\hline region & 6 & 751 & 39 & $7.9^{*} 10^{7}$ & 521 \\
\hline technology & 3 & 801 & 22 & $3.6^{*} 10^{1}$ & 241 \\
\hline tech_capab- & 6 & 202 & 2 & 1 & 130 \\
\hline ility & 13 & 806 & 22 & $1.6^{*} 10^{19}$ & $\begin{array}{l}661(+4 \% \\
\text { timeouts) }\end{array}$ \\
\hline sector & 53 & 929 & 7 & $2.1^{*} 10^{12}$ & $\begin{array}{l}91(+25 \% \\
\text { timeouts) }\end{array}$ \\
\hline state & 53 & & & &
\end{tabular}

Table 8: Runtime analysis of a join of a table with a complete dimension table. Each time, 1000 completeness patterns are used.

Join of Fact Tables. In our second scenario we model a join between two tables that are partially complete by a selfjoin of the network table. We used 50-150 randomly chosen patterns among the 1000 ones that were generated as shown earlier. The results in Table 9 show that also in this case, the number of patterns in the output does not blow up exponentially. Rather, the results show that while the number of completeness patterns grows quadrati- 


\begin{tabular}{|c|c|c|c|c|}
\hline Query & $\begin{array}{l}\text { Query } \\
\text { runtime (ms) }\end{array}$ & $\begin{array}{c}\text { Metadata } \\
\text { computation } \\
\text { runtime }(\mathrm{ms})\end{array}$ & $\begin{array}{l}\text { \# records } \\
\text { query result }\end{array}$ & $\begin{array}{l}\text { \# metadata } \\
\text { records } \\
\text { query result }\end{array}$ \\
\hline SELECT $*$ FROM country, city WHERE country.capital=city.name & 30 & 797 & 278 & 10 \\
\hline SELECT * FROM country, school WHERE country.name=school.country & 260 & 409 & 5467 & 9 \\
\hline SELECT $*$ FROM city, school WHERE city.state=school.state & 175000 & 421 & 2958000 & 46 \\
\hline SELECT $*$ FROM country, school WHERE country.capital=school.city & 160 & 397 & 297 & 9 \\
\hline $\begin{array}{l}\text { SELECT } * \text { FROM country, city, school WHERE country.capital=city.name } \\
\text { AND city.state=school.state }\end{array}$ & 2040 & 900 & 25891 & 46 \\
\hline SELECT $*$ FROM city c1, city c2 WHERE c1.name $=\mathrm{c} 2$.name & 4180 & 991 & 145659 & 100 \\
\hline SELECT $*$ FROM school s1, school s2 WHERE s1.name $=$ s2.name & 2540 & 460 & 42282 & 81 \\
\hline
\end{tabular}

Table 7: Result size and compute time for data and metadata for the queries used in Section 4.2

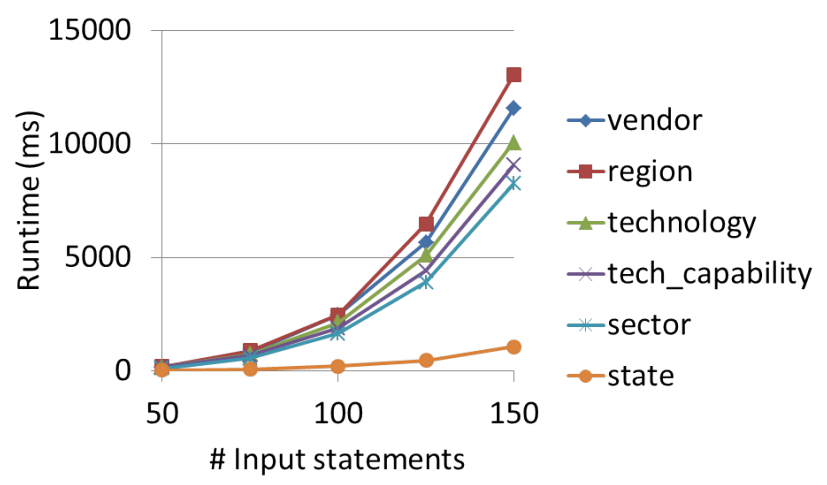

Figure 6: Growth of runtime wrt. number of completeness patterns in a selfjoin, 20 runs per point, with 1000 tuples in the database.

cally before minimization, removal of subsumed patterns (which are subsumed by promoted patterns) gives a final result size that is less than quadratic.

One can also observe a high variation between different attributes. For tech_capability for instance, the minimized output contains only 61 patterns. The reason is that the attribute tech_capability has only three different values, so whenever only two values are present, the chance is high that for those two values completeness patterns exist. Out of the 100 patterns that could be promoted (50 on each side of the join), 61 got promoted, and these 61 patterns then subsume all other patterns that resulted from the regular join.

\begin{tabular}{|c|c|c|c|c|c|}
\hline $\begin{array}{l}\text { Join } \\
\text { attribute }\end{array}$ & $\begin{array}{l}\text { Statem- } \\
\text { ents in } \\
\text { input }\end{array}$ & $\begin{array}{l}\text { Size of } \\
\text { nonmin } \\
\text { imized } \\
\text { output }\end{array}$ & $\begin{array}{l}\text { Size of } \\
\text { minimized } \\
\text { output }\end{array}$ & $\begin{array}{l}\text { Promoted } \\
\text { statements } \\
\text { in minimized } \\
\text { output }\end{array}$ & $\begin{array}{l}\text { Saved } \\
\text { statements } \\
\text { per } \\
\text { promoted } \\
\text { statement }\end{array}$ \\
\hline vendor & 100 & 9,792 & 930 & 232 & 38 \\
\hline region & 100 & 9,499 & 1031 & 224 & 38 \\
\hline technology & 100 & 10,216 & 523 & 236 & 41 \\
\hline tech_capa- & 100 & 10,168 & 420 & 168 & 58 \\
\hline bility & 100 & 6,945 & 932 & 103 & 58 \\
\hline sector & 100 & 2,118 & 467 & 21 & 79 \\
\hline state & 100 & & & \\
\hline
\end{tabular}

Table 9: Growth of completeness patterns in a selfjoin. Notably, the number of patterns in the output does not increase due to promotion but even decreases.

For the state attribute on the other hand, there are 53 values, so any random subset still has a large cardinality and it is unlikely

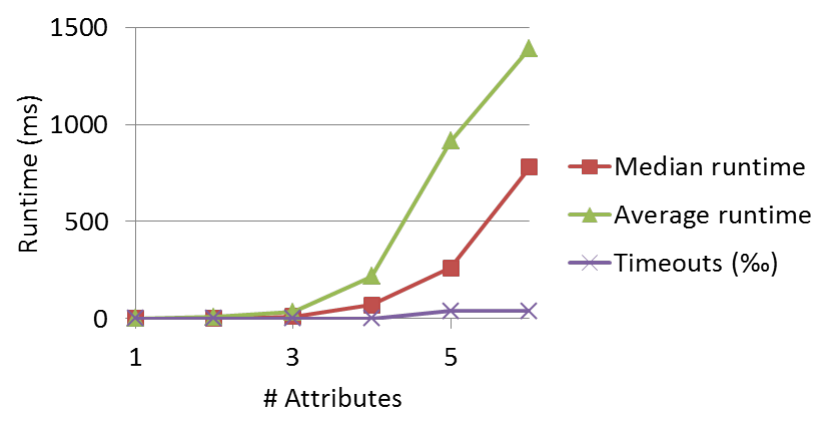

Figure 7: Growth of runtime wrt. number of attributes, 100 runs each using random attribute sets and join values.

that one finds in only 50 patterns enough patterns that together are unifiable for promotion. So as only 17 patterns get promoted, the promoted patterns subsume only few patterns from the regular join and therefore the minimized result has the highest cardinality compared with the other join attributes.

We draw three conclusions: First, the runtime in a join of fact tables grows quadratically in the number of patterns for the input tables (Fig. 6). Second, the runtime grows polynomially wrt. the number of attributes used (Fig. 77). Third, promotion leads to a significant reduction of the number of patterns in the output (Table 9).

\section{E. TOWARDS ALGEBRAIC COMPLETENESS}

One may wonder whether the pattern algebra extended by the promotion operator is computationally complete. Unfortunately, as shown below, this is still not the case. In this section, we discuss a further extension to the pattern algebra, which introduces additional unsatisfiable patterns in intermediate results, which may be needed for computational completeness.

Recall from Example 8 that completeness patterns can be unsatisfiable. The example was about the fact that after a selection by "specialization = hardware", the resulting table is certainly complete for software teams.

While one might consider such patterns as unintuitive and irrelevant, the following example shows that not producing these patterns may lead to missing relevant conclusions in subsequent operations:

Example 10. Consider $M \bowtie_{M . \text { resp=T.name }} \sigma_{\mathrm{spec}=\text { "hw" }}(T)$ again, the join shown in Table 6 The patterns $(*, \mathrm{C}, *, *, *)$ and $(*, *, *, \mathrm{C}, *)$ seem meaningless, because, due to the completeness pattern $(*, *)$ for $\sigma_{\mathrm{spec}=\mathrm{hw}}(T)$ we know that $\mathrm{A}$ and $\mathrm{B}$ are the only hardware teams, and therefore, the join result cannot contain any rows for team $\mathrm{C}$. We call these patterns therefore zombie patterns. 
Can we just drop these meaningless patterns? The contrary is the case as we can benefit from the introduction of more zombie patterns.

As we know that no results for team $\mathrm{D}$ are possible, we can also introduce the zombie patterns $(*, \mathrm{D}, *, *, *)$ and $(*, *, *, \mathrm{D}, *)$. Now suppose we are computing a join with a table Best_teams, which contains the values $\mathrm{A}, \mathrm{C}$ and $\mathrm{D}$, and which is complete ("**).

If we also computed the zombie patterns for $\mathrm{D}$, we can promote the patterns for $\mathrm{A}, \mathrm{C}$ and $\mathrm{D}$ together to the pattern $(*, *, *, *, *, *)$ for the result. If we did not introduce the pattern for $\mathrm{D}$, we could just compute patterns for $\mathrm{A}$ and $\mathrm{C}$ in the join result.

We conclude from this example that information about values that cannot occur in results may be needed to ensure computational completeness.

In this section we discuss how zombie patterns can help deduce more general and thus stronger completeness patterns, how they can be represented, and what is the experimental impact of generating them

Zombie Patterns. Zombie patterns are explicit assertions of completeness for values that can currently not appear in the result of an expression, either due to algebra operations or due to the state of the database instance.

Naively, that is, without further domain information, this approach may require one to assert one zombie pattern for every value in the active domain of the database (set of all constants appearing in the database). Therefore, zombie pattern generation is only feasible for attributes with known domain (e.g. month or state).

\section{E.1 Adding Zombie Patterns}

Zombie patterns naturally arise in two operations: Selection by constant and join. In the first case, the introduction is instanceindependent, in the second case not.

Consider first a selection by constant $\tilde{\sigma}_{A=d}(P)$, where the attribute $A$ has the domain $\operatorname{dom}(A)$. Then, we add the zombie patterns

$$
\{(c, *, \ldots, *) \mid c \in \operatorname{dom}(A), c \neq d\},
$$

to the result of the selection, where we assumed without loss of generality that $A$ is the first attribute of $P$.

For a join, the added patterns depend on the database instance. Consider the join $E_{1} \hat{凶}_{A=B} E_{2}$ of expression $E_{1}$ and $E_{2}$ whose complete parts are described by the pattern sets $P_{1}$ and $P_{2}$. We define the zombie patterns similarly to the promotion result using a function addZombies, which, too, is called twice with interchanged arguments.

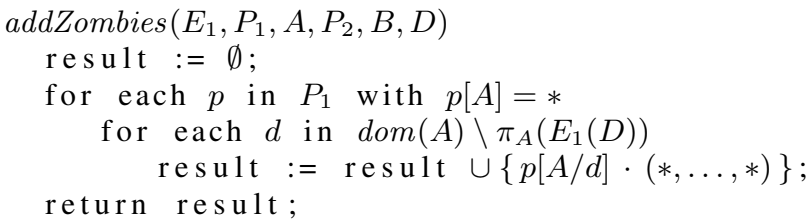

Intuitively, given a completeness pattern with $*$ for the join attribute on one side of the join, we insert all values that are not covered by this pattern into the join position, and extend the pattern with $(*, \ldots)$ on the other side, because no such rows can occur at all due to the completeness pattern.

How many patterns get created depends on the size of the domains and on the database instance. In the next section, we report insights from our use case.

Whether the zombie algebra is computationally complete is an open question to date, because it is not clear whether the extensions

\begin{tabular}{|c|c|c|c|c|}
\hline $\begin{array}{c}\text { Join } \\
\text { attribute }\end{array}$ & $\begin{array}{l}\text { Number of } \\
\text { distinct } \\
\text { attribute } \\
\text { values }\end{array}$ & $\begin{array}{l}\text { Zombie } \\
\text { statements } \\
\text { before mi- } \\
\text { nimization }\end{array}$ & $\begin{array}{l}\text { Zombie } \\
\text { statements } \\
\text { after mi- } \\
\text { nimization }\end{array}$ & $\begin{array}{l}\text { Size of } \\
\text { minimized } \\
\text { output }\end{array}$ \\
\hline vendor & 7 & 6,833 & 380 & 515 \\
\hline region & 6 & 5,841 & 577 & 751 \\
\hline technology & 3 & 2,843 & 516 & 801 \\
\hline tech_capabi- & 6 & 7,001 & 101 & 202 \\
\hline lity & 13 & 12,835 & 510 & 806 \\
\hline sector & 53 & 53,870 & 733 & 929 \\
\hline state & 5 & & \\
\hline
\end{tabular}

Table 10: Number of zombie patterns in a join of a fact table with 1000 patterns with a complete dimension table, before and after minimization.

discussed above introduce all possible kinds of zombie patterns that could be needed in the algebra.

\section{E.2 Impact of Zombie Patterns}

The introduction of zombie patterns gives rise to two questions: The first is how big is the overhead when computing zombie patterns. The second is how often further promotion is possible when computing zombie patterns. We look into both questions next.

Overhead due to Zombie Patterns. The number of zombie patterns generated by selection operations is always the number of possible values of that attribute minus one. In a join, the number of zombie patterns can be varying. We therefore tested both a selfjoin and a join with a complete dimension table, both set up as discussed in Section 5.2

Table 10 contains rows reporting the number of zombie patterns generated in a join of a fact table with 1000 patterns with a complete dimension table, measured before and after removing subsumed patterns from the result. Before minimization there is a clear correlation with the number of attribute values. After minimization, the number of zombie patterns is more uniform around $66 \%$.

In a selfjoin experiment with 100 completeness patterns and 500 random tuples in the database (note that the less data, the more zombie patterns are created), one third of the resulting completeness patterns were zombie patterns.

As minimization of sets of completeness patterns takes a considerable share of the runtime of completeness calculation, the addition of zombie statements also increases the runtime. Both in the selfjoin and in the join with a complete dimension table, the introduction of zombies led to an average runtime increase by $250 \%$.

Additional Inferences Due to Zombie Patterns. Zombie patterns in intermediate join results may be required for promotion in subsequent joins. We therefore computed a three-way-join, where once we computed zombie patterns in the intermediate result and once not. It turned out that out of 200 runs with random sets of 70 completeness patterns for each of the three tables, only in 2 runs there were additional patterns in the result when computing zombie patterns in the intermediate result. In these two runs, once $4 \%$ (10 patterns) and once 7\% (1 pattern) were additionally computed. Over all runs, this amounts to $0.08 \%$ additionally computed patterns. Note that, even though this numbers suggest that zombie patterns have little impact, completeness patterns can have very different generality and a few more computed patterns can still be important in some applications. 